\title{
Effects of Reinforcement Morphology on the Mechanical Behavior of Magnesium Metal Matrix Composites based on Crystal Plasticity Modeling
}

\author{
Jing Zhang ${ }^{1}$, Qiuming Wei ${ }^{2}$ and Shailendra P. Joshi ${ }^{1 *}$ \\ ${ }^{1}$ Department of Mechanical Engineering, National University of Singapore, Singapore 117576 \\ ${ }^{2}$ Department of Mechanical Engineering, University of North Carolina at Charlotte, NC-28223-0001, USA
}

November 26, 2015

${ }^{*}$ Corresponding author: shailendra@nus.edu.sg 


\section{Abstract}

In this work, we investigate the role of elastic inclusions in a plastically anisotropic matrix using crystal plasticity modeling and simulation. Magnesium $(\mathrm{Mg})$ is taken as a model matrix material, which exhibits large plastic anisotropy that originates from slip and twinning mechanisms with dramatically different activation stresses. Using an idealized setup of a periodic unit cell comprising single crystal Mg matrix with an embedded elastic inclusion, we investigate the role of inclusion shape and alignment in the evolution of composite flow responses under compressive and tensile loads. This idealization serves as a model setup for highly textured microstructures that result from extrusion or rolling processes. Detailed analysis reveals how slip and twinning mechanisms evolve with strain and how they depend on the reinforcement morphology. Results indicate that under the loading condition that preferentially activate $\{10 \overline{1} 2\}$ extension twinning, the inclusion morphology and alignment significantly influence the amount of flow hardening at a given strain and its evolution as a function of increasing strain. This twinning induced hardening effect exists in addition to the classical hydrostatic constraint effect induced by the presence of elastically stiff inclusions. We propose a simple empirical expression, which quantifies this coupling. On the other hand, when extension twinning is not an active deformation mechanism, the flow hardening characteristics are similar to the classical MMCs that deform by dislocation slip. We also investigate the effects of reinforcement aspect ratio and volume fraction on the composite responses. Finally, we briefly discuss how these observations on single crystal MMC models can be extended to textured polycrystalline Mg MMCs.

Keywords: Magnesium alloys, Metal Matrix Composites, Strengthening, Anisotropy, Deformation Mechanisms, Crystal Plasticity 


\section{Introduction}

Magnesium $(\mathrm{Mg})$ and Mg-alloys are potential candidates for structural applications ranging from fuel-intensive automotive sector to biomedical components due to attractive properties such as low mass density, high damping capacity and excellent biocompatibility. The Achilles' heel of Mg alloys as preferred structural materials is their lower stiffness and strength relative to their nearest competitor, aluminum (Al) and Al-alloys. Moreover, the hexagonal close-packed crystal structure of $\mathrm{Mg}$ is plastically highly anisotropic, due to which its yield strength $\left(\sigma_{y}\right)$, flow stress $\left(\sigma_{f}\right)$ and ductility are strongly influenced by underlying texture. For single crystal $\mathrm{Mg}$, the ratio of critical activation stresses for the plastically hardest mode and the plastically softest mode can be $\sim 100$ together with dramatically different flow behaviors as well as ductility (Kelley and Hosford, 1968), which can cause issues in processing and manufacturing. There is a growing interest in designing Mg-alloy microstructures with mechanical properties that are tunable based on the expected functionalities. Strategies to mitigate some of these issues include: (i) grain refinement (Yoshida et al., 2003; Yuan et al., 2011), (ii) alloying, (iii) synthesizing composite architectures (Kainer, 1991; Ye and Liu, 2004; Poddar et al., 2007; Habibi et al., 2010; Deng et al., 2012; Shanthi et al., 2012; Shen et al., 2013b) and (iv) texture modification (Young et al., 2007). While each of these approaches can be exploited independently, they are not mutually exclusive (Li and Lavender, 2015). Indeed, it may be beneficial to consider combinations of these strategies in order to design microstructures that provide desired properties. Examples of designed MMC microstructures can be found in the literature (e.g. Clyne and Withers (1995); Rabinovitch et al. (1983); Wang et al. (2006); Li and Lavender (2015)). For instance, it is possible to design Mg matrix nano-composites with desired texture, which result in concurrent enhancement of strength and ductility (Habibi et al., 2010, 2012). Further, by choosing appriopriate alloying elements, such architectures can exploit different strengthening (and sometimes, softening) mechanisms in mitigating the tension-compression strength differential.

The broader focus of this paper is on the mechanics of $\mathrm{Mg}$ metal matrix composites (MMCs). Several experimental reports indicate the effects of reinforcement size and volume fraction on the mechanical behavior of Mg MMCs (Chowdhury et al., 2010a,b; Deng et al., 2010; Hu et al., 
2010; Lelito et al., 2012; Wang et al., 2012; Meixner et al., 2011). Often, adding second phase particles to $\mathrm{Mg}$ matrix strengthens either the tensile or the compressive responses, but seldom both. Even if both the strengths are enhanced, they usually do not address the issue of strength differential. Furthermore, strength enhancement is usually concomitant with a reduction in the strain to failure, barring few exceptions (Habibi et al., 2010, 2012). The strong plastic anisotropy of the basal, non-basal (pyramidal $\langle a\rangle$, prismatic $\langle a\rangle$ and pyramidal $\langle c+a\rangle$ ) slip modes and the presence of twinning $(\{10 \overline{1} 2\}$ extension twinning $(\mathrm{ET})$ and $\{10 \overline{1} 1\}$ contraction twin $(\mathrm{CT}))$ results in highly asymmetric stress-strain characteristics. The presence of hard, elastic reinforcements further complicates the situation by introducing complex stress states (Inem and Pollard, 1993; Szaraz et al., 2007; Deng et al., 2012). Chua et al. (1999) observed that the $0.2 \%$ yield strength (YS), ultimate tensile strength (UTS) and ductility for $\mathrm{Mg}$ alloy with 10 volume percent $\mathrm{SiC}$ reinforcement (size from 15 to $50 \mu \mathrm{m}$ ) were all lower than their un-reinforced Mg-alloy counterparts. On the other hand, Rauber et al. (2011) showed improvement in the YS and UTS with SiC volume fraction increasing from 5\% to 30\%. For extruded Mg MMCs, Garcés et al. (2005) reported decrease in the tension-compression asymmetry ratio with increasing volume fraction of $\mathrm{SiC}_{\mathrm{p}}$ from $5 \%$ to $13 \%$. They indicated that the matrix texture dominated the response in comparison to the strengthening effects due to grain size and load transfer from the matrix to the reinforcement. They also reported weakening of the basal texture with increasing reinforcement volume fraction. More recently, Garcés et al. (2012) also reported systematic reduction in ET activity with increasing reinforcement volume fraction for similar Mg MMCs.

These complex and at times seemingly paradoxical experimental observations are attributed to the interactions between the deformation mechanisms in $\mathrm{Mg}$, which depend on texture and reinforcement details. However, a clear understanding of how reinforcement morphology affects the deformation micromechanics in $\mathrm{Mg}$ matrix is missing, which calls for systematic investigations. To this end, computational investigations that adopt accurate description of HCP plasticity can provide fundamental insights into the micromechanics of Mg MMCs. Motivated by these objectives, we performed computational investigations of idealized Mg MMC microstructures using a crystal plasticity model (Zhang and Joshi, 2012). In this paper, we present the results of that investigation. In particular, 
we elaborate on the effects of the reinforcement shape and alignment on: (i) the macroscropic flow response, (ii) the micromechanics of flow hardening and (iii) the tension-compression flow stress asymmetry.

In the next section, we discuss the details of finite element modeling, simulation set up and material model that was adopted in our investigation.

\section{Details of Mg MMC Models}

The microstructural design variables that influence the mechanical behavior of an MMC are: (i) matrix texture; (ii) matrix grain size, (iii) reinforcement size, (iv) reinforcement volume fraction, (vi) reinforcement shape, (v) reinforcement alignment, and (vi) reinforcement distribution. Obviously, considering all these design variables in a single analysis is a formidable task. In order to keep the problem tractable, we made the following assumptions in setting up the simulation models. First, we considered two relatively dilute reinforcement volume fractions, $f_{r}=0.05$ and 0.10 . Second, we ignored the effects of the matrix grain size and reinforcement size. Finally, we considered only regular arrangement of reinforcements thereby avoiding complicating effects of reinforcement clustering. In the following, we briefly discuss considerations pertaining to matrix texture, reinforcement shape and its alignment with respect to crystallographic orientation.

\subsection{Matrix texture}

Given the strong orientation dependence of $\mathrm{Mg}$ stress-strain characteristics, it is expected that any strengthening due to reinforcement addition will be influenced by the underlying texture. While it will be useful to consider representative volume elements (RVE) comprising textured polycrystalline matrix with embedded inclusions, such an exposition is computationally prohibitive at this stage, especially from the viewpoint of capturing both the micromechanics and its macroscopic effects. We considered a computationally simple alternative, which is a unit cell comprising a single crystal with an embedded inclusion, subjected to periodic boundary conditions (b.c.s). In addition, we only considered situations where these unit cells were loaded perpendicular to the c-axis in tension or in 
compression. This invariably places some constraints: it ignores the influence of grain boundaries that exist in real polycrystals. The perfect normality of the single-crystal c-axis with the loading direction inhibits basal slip that is likely to exist in real polycrystals due to c-axis misorientations in individual grains vis-á-vis macroscopic loading direction. Also, it excludes the possibility of modeling Mg MMCs with random textures. Notwithstanding these limitations, the idealized setup is expected to mimic mechanical attributes of certain Mg MMCs reasonably well because: (i) it broadly mimics extrusion type texture of polycrystalline $\mathrm{Mg}$ where the c-axes of a large fraction of grains are oriented roughly perpendicular to the extrusion direction (Fig. 1), (ii) the kinematic boundary conditions (b.c.'s) prescribed on the unit cells simulate the effects from neighboring inclusions, and (iii) the crystal plasticity modeling approach which is employed here has been shown to predict the micromechanics of Mg and its alloys well (Zhang and Joshi, 2012; Selvarajou et al., 2014). Moreover, this setup serves as an idealization for polycrystalline MMCs hosting inclusions that are smaller than the grain size.

\subsection{Reinforcement shape and alignment}

In MMCs, particulates and short fibers (or whiskers) are common reinforcement shapes; in some cases, disk-shaped reinforcements may also be adopted (Luthringer et al., 2012). In this work, we consider three idealized shapes - spheres, rods and platelets. From a microstructural design viewpoint, it is useful to investigate the effect of reinforcement alignment on the strengthening in Mg MMCs. While this is not relevant for spherical reinforcement, we demonstrate that it has non-trivial consequences in the case of rod and platelet-shaped reinforcements. We consider two canonical orientations for the rod-shaped reinforcements (Fig. 2): (a) Rod-c: rod axis parallel to the crystal c-axis, and (b) Rod-b: rod axis aligned with the basal plane. As shown in the figure, similar orientations arise for platelet-shaped inclusions (respectively, Platelet-c and Platelet- $b$ ). 


\subsection{Three-dimensional Finite Element Modeling}

Figure 3 shows an illustration of an $\mathrm{Mg} \mathrm{MMC}$ with regularly arranged spherical inclusions and the three-dimensional (3D) unit cell extracted from it subject to the following boundary conditions (b.c.'s) - The vertical displacement of the bottom surface is fixed and the top face is subjected

to a uniform velocity $(\dot{\delta})$. The effect periodicity of the neighboring cells is taken into account by constraining the $x_{2}$ and $x_{3}$ faces to remain straight during deformation (hereafter referred to as side-constraint), although they may undergo translation normal to the plane. Although these do not constitute fully periodic boundary conditions (full PBC), our analysis shows that the results presented in this work are qualitatively similar to the full PBC scenario. The interface between the matrix and reinforcement is assumed to remain perfectly bonded during loading. The finite element (FE) simulations are performed using ABAQUS/ STANDARD (Systèmes Dassault, 2012). A typical unit cell is discretized using a fine mesh of C3D4 FEs. The matrix is modeled using single crystal finite deformation plasticity that incorporates slip and twinning (discussed briefly in the next sub-section). The elastic response of $\mathrm{Mg}$ single crystals is transversely elastic with the following elastic constants (in GPa): $C_{11}=59.4, C_{12}=25.61, C_{13}=21.4, C_{33}=61.6$ and $C_{44}=16.0$. The reinforcement is assumed to be isotropic and linearly elastic with Young's modulus $E=375$ GPa and Poisson's ratio $\nu=0.22$.

\subsection{Single crystal plasticity model for $\mathrm{Mg}$}

As noted in the previous section, the kinematics and kinetics of plasticity for Mg matrix are modeled using the user-defined material subroutine facility (UMAT) in ABAQUS/ STANDARD (Zhang and Joshi, 2012). The spatial velocity gradient $\mathbf{L}$ is additively decomposed into the elastic part $\left(\mathbf{L}^{e}\right)$ and plastic part $\left(\mathbf{L}^{p}\right)$

$$
\mathbf{L}=\dot{\mathbf{F}} \mathbf{F}^{-1}=\mathbf{L}^{e}+\underbrace{\mathbf{F}^{e} \tilde{\mathbf{L}}^{p}\left(\mathbf{F}^{e}\right)^{-1}}_{\mathbf{L}^{P}}
$$

where $\dot{\mathbf{F}}$ and $\mathbf{F}^{-1}$ are respectively the material derivative and inverse of the deformation gradient $\mathbf{F}$. The plastic part of velocity gradient in the intermediate configuration is related to the plastic strain 
rate on each slip and twin system via

$$
\mathbf{L}^{p}=\underbrace{\left(1-\sum_{\beta}^{N_{t w}} \xi^{\beta}\right) \sum_{\alpha=1}^{N_{s}} \dot{\gamma}^{\alpha}\left(\boldsymbol{s}^{\alpha} \otimes \boldsymbol{m}^{\alpha}\right)}_{\text {slip in matrix }}+\underbrace{\sum_{\beta=1}^{N_{t w}} \dot{\gamma}^{\beta}\left(\boldsymbol{s}^{\beta} \otimes \boldsymbol{m}^{\beta}\right)}_{\text {twinning in matrix }}+\underbrace{\sum_{\beta=1}^{N_{t w}} \xi^{\beta} \sum_{\bar{\alpha}}^{N_{s}} \dot{\gamma}^{\bar{\alpha}}\left(\boldsymbol{s}^{\bar{\alpha}} \otimes \boldsymbol{m}^{\bar{\alpha}}\right)}_{\text {slip in twinned region }}
$$

where $\boldsymbol{s}^{i}$ and $\boldsymbol{m}^{i}$ respectively represent the direction and normal for $i^{\text {th }}$ slip or twin system, $\xi^{\beta}$ is the twin volume fraction on $\beta$ twin system at time $t$. The model comprises thirty deformation systems (Table 1). The physical basis of the constitutive laws for slip rates $\left(\dot{\gamma}^{\alpha}, \dot{\gamma}^{\bar{\alpha}}\right)$ and twin volume fraction evolution $\dot{\xi}^{\beta}$ can be found in Zhang and Joshi (2012). Here, we briefly summarize the key expressions for completeness.

1. Slip: The slip rate $\dot{\gamma}^{i}$ on the $i^{\text {th }}$ slip system is described by a visco-plastic power law:

$$
\dot{\gamma}^{i}=\dot{\gamma}_{\circ}\left|\frac{\tau^{i}}{g^{i}}\right|^{m} \operatorname{sgn}\left(\tau^{i}\right) \quad(i=\alpha \text { or } \tilde{\alpha})
$$

where $\dot{\gamma}_{0}$ is the reference slip rate, $\tau^{i}=\boldsymbol{m}^{i} \cdot \boldsymbol{\sigma} \cdot \boldsymbol{s}^{i}$ is the resolved shear stress obtained from the Cauchy stress $(\boldsymbol{\sigma}), g^{i}$ is the current strength of the the $i^{t h}$ slip system and $m$ is the rate-sensitivity exponent. The current strength $g^{i}$ of the $i^{\text {th }}$ slip system is given by

$$
g^{i}=\tau_{\circ}^{i}+\int_{t_{\circ}}^{t_{i}}\left(\dot{g}_{s l-s l}^{i}+\dot{g}_{t w-s l}^{i}\right) \mathrm{d} t
$$

where $\tau_{0}^{i}$ is the CRSS, $\dot{g}_{s l-s l}^{i}$ is the hardening due to slip-slip interactions and $\dot{g}_{t w-s l}^{i}$ is the hardening due to twin-slip interactions. The evolution of slip resistance on the $i^{\text {th }}$ slip system due to slip-slip interactions is described as

$$
\dot{g}_{s l-s l}^{i}=\sum_{j=1}^{N_{s}} h_{i j}(\bar{\gamma}) \dot{\gamma}^{j}
$$

where $h_{i j}$ are the self $(i=j)$ and the latent $(i \neq j)$ hardening moduli in the parent or twinned 
regions and

$$
h_{i j}= \begin{cases}h(\bar{\gamma}) & (i=j) \\ q h(\bar{\gamma}) & (i \neq j)\end{cases}
$$

where $1 \leq q \leq 2$ signifies latent hardening; for simplicity, we take $q=1$. The hardening functions for the basal and non-basal slip modes are

$$
h(\bar{\gamma})= \begin{cases}h_{\circ}, & \text { (basal slip) } \\ h_{\circ}^{i} \operatorname{sech}^{2}\left|\frac{h_{\circ}^{i} \bar{\gamma}}{\tau_{s}^{\mathrm{i}}-\tau_{\circ}^{\mathrm{i}}}\right|, & \text { (non-basal slip) }\end{cases}
$$

where $h_{0}^{i}$ is the initial hardening modulus and $\tau_{s}^{i}$ is the saturation stress of the $i^{\text {th }}$ slip system and $\bar{\gamma}$ is the cumulative slip.

2. Twinning: Assuming Schmid behavior, the evolution of ET and CT volume fractions in the parent region is given by

$$
\dot{\xi}^{\beta}=\left\{\begin{array}{l}
\dot{\xi}_{e t}^{0}\left(\frac{\tau^{\beta}}{s_{e t}^{\beta}}\right)^{m_{t}} \\
\dot{\xi}_{c t}^{0}\left(\frac{\tau^{\beta}}{s_{c t}^{\beta}}\right)^{m_{t}}
\end{array}\right.
$$

where $\dot{\xi}_{\text {et }}^{0}$ and $\dot{\xi}_{c t}^{0}$ are the average reference twin volume fraction evolution rates of ET and $\mathrm{CT}$, respectively, $\tau^{\beta}$ is the resolved shear stress, $s^{\beta}$ is the current strength of the the $\beta^{\text {th }}$ twin system and $m_{t}$ is the rate-sensitivity exponent for twinning. The rate of plastic shear $\dot{\gamma}^{\beta}$ is

$$
\dot{\gamma}^{\beta}=\dot{\xi}^{\beta} \gamma^{t w}
$$

where $\gamma^{t w}$ is the twinning shear and is equal to 0.129 and 0.138 for ET and CT, respectively. The current strength of the $\beta^{\text {th }}$ twin system is given by 


$$
s^{\beta}=\tau_{\circ}^{\beta}+\int_{t_{\circ}}^{t_{i}}\left(\dot{s}_{t w-t w}^{\beta}+\dot{s}_{s l-t w}^{\beta}\right) \mathrm{d} t
$$

where $\tau_{0}^{\beta}$ is CRSS of the twin system $\beta, \dot{s}_{t w-t w}^{\beta}$ is the hardening due to twin-twin interactions and $\dot{s}_{s l-t w}^{\beta}$ is the hardening due to slip-twin interactions. We assume that the ET system hardening due to twin-twin interaction follows a saturation type response

$$
\dot{s}_{t w-t w}^{\beta}=h_{\mathrm{et}}^{\beta} \operatorname{sech}^{2}\left|\frac{h_{\mathrm{et}}^{\beta} \bar{\gamma}_{e t}}{\tau_{s_{-} e t}^{\beta}-\tau_{\mathrm{o}_{-} e t}^{\beta}}\right| \dot{\gamma}^{\beta}
$$

where $h_{e t}^{\beta}$ is the initial hardening modulus and $\tau_{s_{-} e t}^{\beta}$ is the saturation stress for ET. $\bar{\gamma}_{e t}$ is the total shear strain on all ET systems. On the other hand, CT hardening is assumed to be of non-saturation type

$$
\dot{s}_{t w-t w}^{\beta}=H_{c t}\left(\sum_{m=1}^{N_{c t}} \xi^{m}\right)^{b} \dot{\gamma}^{\beta}
$$

where $N_{c t}$ is the total number of CT systems. $H_{c t}$ and $b$ are hardening parameters for CT. Twin system hardening is assumed to be independent of the slip in the crystal $\left(\dot{s}_{s l-t w}^{i}=0\right)$, while twinning is assumed to affect the slip hardening $\left(\dot{g}_{t w-s l}^{i}\right)$ as

$$
\dot{g}_{t w-s l}^{\beta}=\left\{\begin{array}{l}
h_{e t \_s l}^{\beta} \operatorname{sech}^{2}\left|\frac{h_{e t \_s l}^{\beta} \bar{\gamma}_{e t}}{\tau_{s_{-} e t}^{\beta}-\tau_{o_{-} e t}^{\beta}}\right| \\
0.5 H_{c t \_s l}\left(\bar{\gamma}_{c t}\right)^{-0.5} \dot{\gamma}_{c t},
\end{array}\right.
$$

For a given microstructure, the relative importance of the different deformation modes at any given strain is quantified using

$$
\bar{\Gamma}^{\alpha}=\frac{1}{V} \int \eta^{\alpha} d v
$$

where $\bar{\Gamma}^{\alpha} \leq 1$ is the volume-averaged relative activity of the $\alpha^{\text {th }}$ slip or twin mode at each Gauss point, $V$ is the matrix volume and $d v$ is the volume represented by a Gauss point. For $\alpha^{\text {th }}$ mode, 
the relative activity $\eta^{\alpha}$ at each Gauss point is given by

$$
\eta^{\alpha}=\frac{\sum_{i=1}^{n} \xi_{\alpha}\left|\Delta \gamma_{i}^{\alpha}\right|}{\sum_{\alpha=1}^{m} \sum_{i=1}^{n} \zeta_{i}\left|\Delta \gamma_{i}^{\alpha}\right|} ; \quad \zeta_{i}=\left\{\begin{array}{cr}
1-\sum_{\beta=1}^{N_{t w}} \zeta^{\beta} & \text { for slip in matrix } \\
1 & \text { for twin in matrix } \\
\xi^{\beta} & \text { for slip in twin }
\end{array}\right.
$$

where $n$ is the number of slip or twin systems for a given deformation mode under consideration (Table 1 ) and $m=6$ is the number of types of deformation modes ( 4 slip and 2 twin modes, Table $1)$.

The HCP-CP model is implemented as a User MATerial (UMAT) subroutine ABAQUS/STANDARD ${ }^{\circledR}$ (Systèmes Dassault, 2012). The material parameters in Eq. (3) through (13) are consolidated in Table 2.

\section{Results and Discussion}

In this section, we present the results of the 3D crystal plasticity simulations of Mg MMCs subjected to tensile and compressive loading, including the overall responses and their micromechanical underpinnings. The results and the discussion in this section correspond to fixed reinforcement volume fraction $\left(f_{r}\right)$ equal to $5 \%$. Further, for the rod-shape reinforcement we set the length $(l)$ to diameter $(d)$ ratio equal to 2 and for the platelet-shaped reinforcement the thickness $t_{p}$ is taken equal to $d_{p} / 5$ where $d_{p}$ is the platelet diameter. Section 4.1 discusses the effects of $f_{r}$ (for fixed aspect ratio) and aspect ratio (for fixed $f_{r}$ ).

\subsection{Overall responses: effect of reinforcement shape and alignment}

Figure 4 shows the overall compressive and tensile stress-strain responses of the simulated MMCs as functions of the reinforcement shape and alignment. As expected, even a dilute $f_{r}$ causes strengthening of both tensile and compressive flow responses relative to monolithic Mg. A highlight of this result is that the reinforcement shape and alignment significantly influence the amount of flow hardening, especially in the compressive response. It is interesting to note that while monolithic 
$\mathrm{Mg}$ shows the characteristic sigmoidal response in compression that results from ET activation, $\mathrm{Mg}$ MMCs tend to vary between mildly sigmoidal to concave depending upon the reinforcement type and alignment. Some of the salient macroscopic characteristics are:

1. Of all the cases considered here, Platelet- $c$ and Rod- $b$ reinforcements give rise to the strongest hardening in both tension and compression. Further, they exhibit nearly identical overall responses, although Platelet- $c$ tends to provide somewhat higher hardening in the small-tomoderate strain regime;

2. Platelet- $b$ exhibits high initial tensile and compressive hardening similar to that of Platelet$c$, however its compressive hardening becomes progressively weaker with strain. We faced convergence issues in the tensile simulation for Platelet- $b$ case because of which the simulation could not be performed over a larger strain range;

3. The tensile as well as compressive hardening of the Rod-c MMCs is significantly weaker compared to the Rod-b and Platelet-c cases;

4. Rod-c and Sphere exhibit nearly identical flow responses in tension. In compression, the compressive behavior of Rod-c is much weaker than that of Sphere at larger strains, and

5. The compressive hardening response of Sphere MMC qualitatively resembles the monolithic $\mathrm{Mg}$.

Based on above observations, we infer that the Mg MMCs with platelet-shaped and rod-shaped reinforcements that are aligned with the basal plane provide the best strengthening in both compression and tension. Naturally, the higher the volume fraction of a given reinforcement type, the stronger will be the composite response.

For the matrix crystallographic orientation considered here (Fig. 2), initial compressive response is dictated by extension twinning, which is the second softest plastic deformation mode in $\mathrm{Mg}$ after the basal slip. On the other hand, the tensile response is governed by non-basal slip and contraction twin modes. As seen in Fig. 4, the effect of reinforcement on the compressive flow response is 
more remarkable compared to the tensile response. It is important to ascribe the micromechanical attributes to the overall characteristics outlined in the preceding discussion. In the following, we discuss these results from the viewpoints of the deformation modes and constraint effects.

It is useful to discuss the result in Fig. 4 from the viewpoint of tension-compression asymmetry. We define the asymmetry ratio as $\mathrm{AR}=\sigma_{c} / \sigma_{t}$ where $\sigma_{c}$ and $\sigma_{t}$ are the flow stresses in compression and tension, respectively, at fixed overall strain. Figure 5 shows the evolution of AR as a function of the nominal strain. With the addition of reinforcement the AR is reduced, but the extent of reduction is influenced by the reinforcement shape and alignment. At small strains, the rod-shaped and platelet-shaped reinforcements provide better reduction in AR than spherical reinforcements. Interestingly, Robson et al. (2011) drew similar conclusions from their analysis of Mg alloys reinforced by nano-scale precipitates, although the governing mechanisms for strengthening are different from those in the present scenario. With increasing strain, AR approaches one indicating that the magnitudes of tensile and compressive hardening responses tend to become similar. An overall reduction in the plastic flow asymmetry at a given strain may result from: $(i)$ enhancement in $\sigma_{c}$ with relatively smaller/ no enhancement in $\sigma_{t}$, or $(i i)$ reduction in $\sigma_{t}$ with/ without increasing $\sigma_{c}$. From Fig. 4 it is evident that both $\sigma_{c}$ and $\sigma_{t}$ increase, but the former increases faster than the latter resulting in an overall reduction of AR. Finally, we also note from Fig. 5 that in some cases (Rod-c, Platelet-c) the AR tends to saturate below 1, which implies that the ratio of the hardening rates in tension and compression reaches a steady state.

\subsection{Micromechanics of strengthening in compression}

In this section, we discuss correlation between different deformation mechanisms and their influence on the composite hardening responses under compressive loading. As compression is applied perpendicular to the c-axis ET is expected to play a dominant role in the matrix behavior. As shown next, the manner in which ET volume fraction evolves is strongly influenced by the reinforcement shape and alignment. 


\subsubsection{Deformation Micromechanics and the Role of Extension Twinning}

In order to explain how slip and twin activities influence the overall Mg MMC responses in Fig. 4, we plot the evolution of relative deformation activities $\left(\bar{\Gamma}^{\alpha}\right)$ as a function of compressive strain. Figure 6 a-d shows the evolution of $\bar{\Gamma}^{\alpha}$ for the Rod-b (strongest response) and Platelet- $b$ (weaker response at larger strain) cases together with those of $\mathrm{Mg} M \mathrm{MC}$ with spherical reinforcement and monolithic Mg for comparison.

In monolithic Mg (Fig. 6(d)), the ET mode completely contributes to the plasticity up to strain about 0.06 at which significant crystal reorientation occurs and only then dose slip kick in. In comparison, the presence of reinforcements results in activation of slip modes even at early stages (Fig. 6(a,b,c)). As a result, the relative contribution from ET to the overall plastic strain is lower in MMCs compared to the monolithic Mg.

For the three exemplar MMC cases shown in Fig. 6, the basal and pyramidal $\langle c+a\rangle$ modes are active over a significant range of strain. Although the other modes also show some activity especially at larger strains, they are not as dominant as the pyramidal and basal slip modes. For Platelet- $b$ case the pyramidal $\langle c+a\rangle$ slip exists over almost the entire strain range considered here (Fig. 6b), while it occurs in the Rod-b and Sphere beyond the strain of $\sim 0.04$. However, the pyramidal $\langle c+a\rangle$ slip activity is significantly more dominant in Rod-b and Sphere cases compared to the Platelet-b case. A dramatic increase in the pyramidal $\langle c+a\rangle$ slip is concomitant with the ET induced reorientation (an extreme scenario being that of monolithic Mg, Fig. 6d). From these observations we deduce that the relatively weaker pyramidal $\langle c+a\rangle$ slip activity in Platelet- $b$ MMC manifests as sluggish hardening at larger strains (Fig. 4). That being said, the initial weak hardening for Sphere MMC

compared to Rod- $b$ MMC despite similar relative activity needs to be investigated. As we show later, it is influenced by constraint effects.

Figure 7 reveals the spatial evolution of ET within the matrix volume for three MMC cases. Clearly, the twin distribution and volume at a given strain are influenced by the reinforcement 
shape and alignment; the latter is also reflected in Fig. 8. An important aspect that needs to be pointed out is that in all the cases shown here the matrix volume in vicinity of reinforcement remains untwinned even at $\bar{\varepsilon}=0.07$. This is because of the triaxiality of the stress state around the reinforcement, which will be elaborated in the following discussion.

Figure 8 compares the ET volume fraction evolution with strain for all MMC cases. The Sphere MMC exhibits the highest saturation ET volume fraction while Platelet- $b$ MMC exhibits the lowest saturation twin volume fraction. Although the evolution trends for all cases are qualitatively similar, the evolution rates are quite different. The twin volume fraction begins to evolve earlier $(\bar{\varepsilon} \sim 0.01-0.02)$ in the rod and platelet reinforced MMCs while it starts only at $\bar{\varepsilon} \sim 0.03$ for the Sphere MMC. Correlating Figs. 4 and 8, we note that the MMCs possess the lowest saturated ET volume fractions. (Rod-c and Platelet-b) also exhibit the weakest hardening rates at moderate to large strains. In comparison, the Rod-b and Platelet-c MMCs undergo large amounts of twinning and also possess the highest hardening rates. Finally, although the Sphere MMC results in the highest saturation ET volume fraction amongst all cases, the overall flow stress at any given strain is lower than the Rod-b and Platelet-c MMCs. This is because in the latter cases the ET volume fraction begins to evolve earlier and it continues to evolve over a larger strain range than in the former. From these observations, we infer that along with the total ET volume fraction and the rate of change of ET volume fraction there must be additional factors influencing the MMC hardening, discussed next.

\subsubsection{Quantifying the Roles of Extension Twinning and Hydrostatic Effects on Flow Stress}

It is well known that the load applied to an MMC is transferred to the reinforcing inclusions by the softer metallic matrix thereby producing an overall hardening response. The stronger the constraint exerted by the elastic reinforcement and the prescribed b.c.'s, the stronger is the overall mechanical behavior (Brockenbrough et al., 1991). In addition to this, the ET induced lattice reorientation due to c-axis activates plastically harder modes causing matrix hardening. It is important to quantify 
the effect of this twinning induced hardening and its interaction with the hardening induced by constraint effects from both the prescribed b.c.'s and the reinforcement morphology. To that end, Table 3 compiles the ET volume fraction $\left(\xi_{\mathrm{t}}\right)$, the average hydrostatic stress $\left(\bar{\sigma}_{\mathbf{H}}=\frac{1}{V_{\text {matrix }}} \int \sigma_{h} d V\right.$, where $\sigma_{h}$ is the hydrostatic stress at a Gauss point) and the corresponding flow stress $\left(\bar{\sigma}_{\mathrm{f}}\right)$ at $\varepsilon=0.03$ and 0.07 . It can be seen that at $\varepsilon=0.03$ where the ET volume fraction is small the overall hardening of $\mathrm{Mg}$ MMCs correlates well with $\bar{\sigma}_{\mathrm{H}}$. In other words, the twinning-induced hardening effects do not influence the overall flow stress at small strains (less than the twinning induced macroscopic strain) and is governed by constraint-induced triaxial effects that are modulated by the nature of the reinforcement and the prescribed b.c. This is consistent with previous numerical studies on strengthening of MMCs where twinning is not present (Brockenbrough et al., 1991; Christman et al., 1989; Llorca et al., 1991).

Referring back to Table 3, the constraint effect does not enjoy a correlation with the flow stress at larger strain $(\varepsilon=0.07)$. Note that at $\varepsilon \geq 0.06$, a substantial matrix volume has already undergone ET-induced crystal reorientation, which activates plastically hard modes - pyramidal $\langle c+a\rangle$ slip and CT. The lack of correlation between the constraint effect and the flow stress underscores the role of ET. This is an important observation because, twinning-induced hardening depends on the nature of ET volume fraction evolution, which in turn is influenced by the reinforcement type and alignment (Fig. 8). A rigorous analytical description of the dependence of the flow stress on the combined effect of reinforcement and side constraints, rate of ET and ET volume fraction is not attempted in this paper. However, as shown in Table 4 a simple empirical expression that appears to correlate reasonably well with the flow stress $\left(\bar{\sigma}_{\mathrm{f}}\right)$ predicted by HCP-CP simulations is

$$
\bar{\sigma}_{\mathrm{f}} \approx \mathrm{A} \xi_{\mathrm{t}} \bar{\sigma}_{\mathrm{H}}^{\prime}
$$

where $\xi_{\mathrm{t}}$ is the ET volume fraction at a given $\bar{\varepsilon}$, and $\bar{\sigma}_{\mathrm{H}}^{\prime}=\mathrm{d} \sigma_{\mathrm{H}} / \mathrm{d} \bar{\varepsilon}$. The pre-factor A, which may be considered as an indicator of the coupling between ET and the hydrostatic constraint effect is $0.25 \pm 0.016$. While Eq. (16) is simplistic, it could serve as a preliminary basis to substantiate the role of deformation twinning in the overall MMC hardening. 


\subsection{Micromechanics of strengthening in tension}

As noted earlier, the tensile stress-strain responses in Fig. 4 exhibit an enhancement in the flow stress, which depends on the geometry and alignment of reinforcement. Figure 9 shows the relative activity plots for MMCs with rod-shaped and spherical reinforcements along side the monolithic Mg. Although not included here, the relative activity plots for MMC with platelet-shaped reinforcements as they are similar to those of the Rod-b case; the Rod-c case is similar to Sphere MMC. It is clear that irrespective of the reinforcement type and alignment, the presence of an elastic inclusion results in a larger contribution of the plastically hard pyramidal $\langle c+a\rangle$ slip mode. In comparison, the pyramidal $\langle c+a\rangle$ activity is much weaker in monolithic $\mathrm{Mg}$ and it occurs only at much higher strains. In all the cases, there is some activity on the contraction twin (CT) systems, which is also a plastically hard mode, although its importance tends to drop with progressive deformation because of the strong CT-CT interaction embedded in the constitutive model (Zhang and Joshi, 2012). A primary difference between the relative activities under tensile loading as compared to those under compressive loading is that, unlike in the latter scenario the activated slip modes under tensile loading are not effected by ET. The varying degree of hardening of the tensile flow response despite similar relative slip activities is attributed to the hydrostatic constraint from the elastic inclusion and b.c.'s (Brockenbrough et al., 1991).

A quantitative understanding of the reinforcement shape and alignment on matrix field quantities and the related strain hardening responses of the MMCs can be obtained from Fig. 10. This is a plot of the matrix volume fraction in the RVE, which has the magnitude of the average hydrostatic stress $\left(\left|\sigma_{h}\right|\right)$ greater than the value indicated on the abscissa at a fixed far-field tensile strain (Brockenbrough et al., 1991). As seen in the plot, although $\left|\sigma_{h}\right|$ for different cases is in the similar range, Platelet- $c$ and Rod-b MMCs possess a somewhat larger fraction of the matrix that experiences a higher hydrostatic stress compared to the Sphere and Rod-c cases. Consistent with this, the overall ranking of the flow stress is as follows (Fig. 4 at $\bar{\varepsilon}=0.05):\{$ Platelet- $c \approx$ Rod-b $\}>\{$ Rod-c $\approx$ Sphere\}.

While Fig. 9 gives the relative importance of various deformation modes in the total plastic 
deformation, it is useful to inspect the distribution of the accumulated plastic shear strain $\left(\sum_{\alpha} \gamma^{\alpha}\right)$. Figure 11 compares the distribution of the accumulated plastic shear strain at $\bar{\varepsilon}=0.05$ in the middle section parallel to the loading direction for the strong (Rod-b) and comparatively softer (Sphere) responses. We note that plastic deformation is more heterogeneous in the Rod-b MMC, which also implies stronger strain gradients. Although not considered in this work, they may play an important role in overall flow hardening, especially in sub-micron sized reinforcements.

\section{Further Results and Trends}

In the preceding sections, we focused on the effects of reinforcement shape and alignment keeping the aspect ratio and volume fraction constant. It is natural to ask - how would changing aspect ratio or the reinforcement volume fraction affect the responses? In the following, we briefly report the trends for a limited number of scenarios. We also present a preliminary analysis on how the present results on MMC composite with single crystal matrix may be extended to polycrystalline architectures.

\subsection{Reinforcement aspect ratio}

From the preceding discussions, it is clear that the MMC with Rod-b and Platelet-c type reinforcements result in the best overall tensile and compressive responses. Therefore, it is instructive to study the effect of the reinforcement aspect ratio on the response enhancement. For brevity, we restrict the discussion to the Rod-b MMC as similar observations hold for the Platelet-c case as well. Figure 12 compares the compressive and tensile responses of Rod-b MMC for aspect ratios 2 and 3 for fixed reinforcement volume fraction $\left(f_{r}=0.05\right)$. As seen, a longer rod-shaped reinforcement

aligned with the loading direction provides stronger tensile and compressive responses (Fig. 12). This is expected because the longer rod-shaped reinforcement mimics the case of a continuous fiber (or nearly so), which results in a dominant portion of the total load being shared by the reinforcement.Figure.12(b) indicates that increased aspect ratio of the reinforcement also reduces the tension-compression asymmetry. 


\subsection{Effect of reinforcement content on compression-tension asymmetry}

Figure 13 indicates that increasing reinforcement volume fraction reduces the compression-tension asymmetry of Rod-b MMCs. Simulations indicate enhanced strengthening of composites with increasing particle content under both tension and compression. This observation is consistent with experimental reports (Ye and Liu, 2004; Manoharan et al., 2002; Nie et al., 2011). The same trend is also revealed for aluminum-matrix composites. However, texture and grain size may be influenced by the amount of reinforcements in composites(Ye and Liu, 2004; Garcés et al., 2005). In such situations, the influencing factors will interact in a complicated manner. On the other hand, if the initial texture remains unchanged with increasing reinforcement volume fraction (as assumed in this work) then the higher the reinforcement volume fraction the stronger the composite responses under both tension and compression. This is consistent with the results in Garcés et al. (2012) where the extruded textures of $\mathrm{AZ31}-5 \% \mathrm{SiC}$ and $\mathrm{AZ31}-10 \% \mathrm{SiC}$ were similar.

\subsection{Texture effect on tension-compression asymmetry reduction}

For a polycrystalline metal, the Taylor factor $(M)$ is commonly used to relate the macroscopic yield stress $\sigma_{y}$ with the critical resolved shear stress (CRSS) of the dominant deformation mode $\left(\tau_{0}\right)$ for single crystal. In conventional cases of BCC and FCC metals, $M$ is usually taken to be 3.0 for random texture (Kocks, 1970). However, it was shown that $M$ is $\sim 5.0$ for polycrystalline titanium of random texture, and it actually has a range of value depending on the actual slip modes responsible for plastic deformation (Conrad, 1973). Naturally, the specific value of $M$ is a function of crystal structure and texture of the polycrystalline system. It embeds texture related information of the polycrystal (Cáceres and Lukáč, 2008). In this context, recently Shen et al. (2013a) defined an analogous parameter

$$
\Pi=\frac{\sigma_{y}}{\sum \bar{\Gamma}^{\alpha} \tau_{\alpha}}
$$

where $\tau_{\alpha}$ is the CRSS of the $\alpha^{\text {th }} \operatorname{slip} /$ twin mode and $\bar{\Gamma}^{\alpha}$ is the corresponding averaged relative activity (Figs. 6 and 9). Using Eq. (17) the behavior of Mg MMCs under tension and compression 
can be rationalized in terms of the weighted deformation activity. As an illustration, Table 5 summarizes the averaged relative activities of slip and twin modes at $0.5 \%$ strain for Sphere and Rod-b MMCs (from Figs. 6 and 9). Using the CRSS parameters for Mg from Table 1, Table 6 reports the values of $\Pi$ from Eq. (17). For the Rod-b Mg MMCs, the ratio $\bar{\Pi}=\Pi_{\text {compression }} / \Pi_{\text {tension }}$ is $\sim 2$, which coincides with the higher AR (i.e. reduced asymmetry) compared to the monolithic Mg. For the Sphere MMC, for which the AR is much lower (i.e. higher asymmetry), $\bar{\Pi} \sim 1.05$. In other words, a higher asymmetry is tantamount to a lower $\bar{\Pi}$ and vice-versa. Thus, $\Pi$ provides a way to quantify the compression-tension asymmetry from a textural viewpoint.

\section{Conclusion}

In this work, we discuss the effect of reinforcement shape, alignment and volume fraction on the overall response of Mg MMCs unit cells using single crystal plasticity based finite element simulations. The matrix crystal orientation idealizes an extrusion-type texture where the basal planes are roughly aligned perpendicular to the extrusion direction. Our simulations reveal that Mg MMCs with rod shaped inclusions with their axis aligned parallel to the loading direction (Rod-b) and platelet shaped inclusions with their axis aligned perpendicular to the loading direction (Platelet-c) are most effective in improving the strengthening in both compression and tension. Under compression, the flow hardening can be effectively enhanced if ET initiates at early strains, evolves faster with increasing strain, and occupies a large volume of the matrix (80\%). A preliminary analysis suggests that, in the presence of ET the flow stress at a given strain correlates with the product of ET volume fraction and the rate of change of hydrostatic stress (referred to as the constraint effect). At low ET volume fractions the flow stress correlates well with the average hydrostatic stress in the matrix. Under tensile loading, ET is not activated and the flow stress at a given strain correlates well with the average hydrostatic stress in the matrix at that strain.

These effects have a collateral influence on the compression-tension asymmetry. Rod- $b$ and Platelet-c reinforced Mg MMCs exhibit the best reduction in the asymmetry. We discuss the relevance of the reinforcement details on the asymmetry ratio (AR) by connecting its trends with a 
Taylor-like factor $(\Pi)$. The analysis indicates that to reduce the asymmetry while maintaining the tensile yield strength, it is necessary to activate plastically hard modes under compression at yield. This conclusion relates back to the dependence of the flow stress on ET and constraint effects.

Although no quantitative experimental validation of our modeling and simulations for the $\mathrm{Mg}$ based MMCs is possible at this point, we envision that fabrication of prescribed reinforcement shape and orientation in Mg-based MMCs would be plausible. Insights gained from this work should serve as a useful start toward designing Mg MMC architectures with desirable mechanical attributes.

\section{Acknowledgment}

JZ acknowledges financial support from A*STAR-NSF Materials World Network Program (R-265000-373-305). SPJ acknowledges partial support from US ARMY ITC-PAC under research contract \# FA5209-10-P-0047. QW acknowledges the support from US Army Research Laboratory under Contract no. W911NF-14-2-0061. The authors also acknowledge Mr. Balaji Selvarajou for his assistance in performing some FE simulations during manuscript revision.

\section{References}

Brockenbrough, J., Suresh, S., Wienecke, H., 1991. Deformation of metal-matrix composites with continuous fibers: geometrical effects of fiber distribution and shape. Acta Metallurgica et Materialia 39, 735-752.

Cáceres, C., Lukáč, P., 2008. Strain hardening behaviour and the Taylor factor of pure magnesium. Philosophical Magazine 88, 977-989.

Chowdhury, A.S.M.F., Mari, D., Schaller, R., 2010a. The effect of the orientation of the basal plane on the mechanical loss in magnesium matrix composites studied by mechanical spectroscopy. Acta Materialia 58, 2555-2563. 
Chowdhury, A.S.M.F., Mari, D., Schaller, R., 2010b. Thermal stress relaxation in magnesium matrix composites controlled by dislocation breakaway. Composites Science and Technology 70, 136-142.

Christman, T., Needleman, A., Suresh, S., 1989. An experimental and numerical study of deformation in metal-ceramic composites. Acta Metallurgica 37, 3029-3050.

Chua, B., Lu, L., Lai, M., 1999. Influence of sic particles on mechanical properties of Mg based composite. Composite Structures 47, 595-601.

Clyne, T., Withers, P., 1995. An introduction to metal matrix composites. Cambridge University Press.

Conrad, H.and Doner, M.d.M.B., 1973. Deformation and fracture. Titanium Science and Technology 2, 969-1004.

Deng, K., Wang, C., Shi, J., Wu, Y., Wu, K., 2012. Microstructure evolution mechanism of micron particle reinforced magnesium matrix composite at room temperature. Materials Chemistry and Physics 134, 581-584.

Deng, K.K., Wu, K., Wu, Y.W., Nie, K.B., Zheng, M.Y., 2010. Effect of submicron size SiC particulates on microstructure and mechanical properties of AZ91 magnesium matrix composites. Journal of Alloys and Compounds 504, 542-547.

Garcés, G., Onorbe, E., Pérez, P., Klaus, M., Genzel, C., Adeva, P., 2012. Influence of SiC particles on compressive deformation of magnesium matrix composites. Materials Science and Engineering: A $533,119-123$.

Garcés, G., Pérez, P., Adeva, P., 2005. Effect of the extrusion texture on the mechanical behaviour of $\mathrm{Mg}-\mathrm{SiC}_{p}$ composites. Scripta Materialia 52, 615-619.

Habibi, M.K., Gupta, M., Joshi, S.P., 2012. Size-effects in textural strengthening of hierarchical magnesium nano-composites. Materials Science and Engineering: A .

Habibi, M.K., Joshi, S.P., Gupta, M., 2010. Hierarchical magnesium nano-composites for enhanced mechanical response. Acta Materialia 58, 6104-6114. 
Hu, Z., Yan, H., Liu, S., Ling, L., Nie, Q., 2010. Compression deformation behavior of semisolid n-SiCp/AZ61 magnesium matrix composites. Jixie Gongcheng Xuebao/Journal of Mechanical Engineering 46, 28-33.

Inem, B., Pollard, G., 1993. Interface structure and fractography of a magnesium-alloy, metal-matrix composite reinforced with SiC particles. Journal of Materials Science 28, 4427-4434.

Kainer, K., 1991. Influence of heat treatment on the properties of short-fibre-reinforced magnesium composites. Materials Science and Engineering: A 135, 243-246.

Kelley, E., Hosford, W., 1968. Plane-strain compression of magnesium and magnesium alloy crystals. Transactions of the Metallurgical Society of AIME 242, 5-13.

Kocks, U.F., 1970. The relation between polycrystal deformation and single-crystal deformation. Metallurgical Materials Transactions B 1, 1121-1143.

Lelito, J., Zak, P.L., Shirzadi, A.A., Greer, A.L., Krajewski, W.K., Suchy, J.S., Haberl, K., Schumacher, P., 2012. Effect of sic reinforcement particles on the grain density in a magnesiumbased metal-matrix composite: Modelling and experiment. Acta Materialia 60, 2950-2958.

Li, D., Lavender, C., 2015. Strengthening and improving yield asymmetry of magnesium alloys by second phase particle refinement under the guidance of integrated computational materials engineering. Journal of Engineering Materials and Technology 137, 031008.

Llorca, J., Needleman, A., Suresh, S., 1991. An analysis of the effects of matrix void growth on deformation and ductility in metal-ceramic composites. Acta Metallurgica et Materialia 39, $2317-2335$.

Luthringer, B.J., Hort, N., Feyerabend, F., 2012. Metal matrix composites: Magnesium. Wiley Encyclopedia of Composites .

Manoharan, M., Lim, S., Gupta, M., 2002. Application of a model for the work hardening behavior to $\mathrm{Mg} / \mathrm{SiC}$ composites synthesized using a fluxless casting process. Materials Science and Engineering: A $333,243-249$. 
Meixner, M., Fitzpatrick, M., Reimers, W., 2011. Measurement of the evolution of internal strain and load partitioning in magnesium hybrid composites under compression load using in-situ synchrotron X-raydiffraction analysis. Composites Science and Technology 71, 167-176.

Nie, K.B., Wang, X.J., Wu, K., Hu, X.S., Zheng, M.Y., Xu, L., 2011. Microstructure and tensile properties of micro-SiC particles reinforced magnesium matrix composites produced by semisolid stirring assisted ultrasonic vibration. Materials Science and Engineering: A 528, 8709-8714.

Poddar, P., Srivastava, V., De, P., Sahoo, K., 2007. Processing and mechanical properties of SiC reinforced cast magnesium matrix composites by stir casting process. Materials Science and Engineering: A 460, 357-364.

Rabinovitch, M., Stohr, J., Khan, T., Bibring, H., 1983. Directionally solidified composites for application at high temperature. Fabrication of composites(A 84-32826 14-24). Amsterdam, North Holland, 1983, , 295-372.

Rauber, C., Lohmller, A., Opel, S., Singer, R., 2011. Microstructure and mechanical properties of $\mathrm{SiC}$ particle reinforced magnesium composites processed by injection molding. Materials Science and Engineering: A 528, $6313-6323$.

Robson, J., Stanford, N., Barnett, M., 2011. Effect of precipitate shape on slip and twinning in magnesium alloys. Acta Materialia 59, 1945-1956.

Selvarajou, B., Shin, J.H., Ha, T.K., Choi, I.s., Joshi, S.P., Han, H.N., 2014. Orientation-dependent indentation response of magnesium single crystals: Modeling and experiments. Acta Materialia $81,358-376$.

Shanthi, M., Jayaramanavar, P., Vyas, V., Seenivasan, D., Gupta, M., 2012. Effect of niobium particulate addition on the microstructure and mechanical properties of pure magnesium. Journal of Alloys and Compounds 513, 202-207.

Shen, J., Li, Y., Wei, Q., 2013a. Statistic derivation of Taylor factors for polycrystalline metals with application to pure magnesium. Materials Science and Engineering: A 582, 270-275. 
Shen, J., Yin, W., Wei, Q., Li, Y., Liu, J., An, L., 2013b. Effect of ceramic nanoparticle reinforcements on the quasistatic and dynamic mechanical properties of magnesium-based metal matrix composites. Journal of Materials Research 28, 1835-1852.

Systèmes Dassault, 2012. Abaqus 6.12 reference manuals. Dassault Systèmes Simulia Corp. .

Szaraz, Z., Trojanova, Z., Cabbibo, M., Evangelista, E., 2007. Strengthening in a WE54 magnesium alloy containing SiC particles. Materials Science and Engineering: A 462, 225-229.

Wang, W.G., Xiao, B.L., Ma, Z.Y., 2012. Evolution of interfacial nanostructures and stress states in $\mathrm{Mg}$ matrix composites reinforced with coated continuous carbon fibers. Composites Science and Technology 72, 152-158.

Wang, Y., Chang, C., Lee, C., Lin, H., Huang, J., 2006. Texture and weak grain size dependence in friction stir processed $\mathrm{Mg}-\mathrm{Al}-\mathrm{Zn}$ alloy. Scripta materialia 55, 637-640.

Ye, H., Liu, X., 2004. Review of recent studies in magnesium matrix composites. Journal of Materials Science 39, 6153-6171.

Yoshida, Y., Cisar, L., Kamado, S., Kojima, Y., 2003. Effect of microstructural factors on tensile properties of an ECAE-processed AZ31 magnesium alloy: Special issue on platform science and technology for advanced magnesium alloys, ii. Materials transactions-JIM 44, 468-475.

Young, M., DeFouw, J., Almer, J., Dunand, D., 2007. Load partitioning during compressive loading of a $\mathrm{Mg} / \mathrm{MgB}_{2}$ composite. Acta Materialia 55, 3467-3478.

Yuan, W., Panigrahi, S., Su, J.Q., Mishra, R., 2011. Influence of grain size and texture on Hall-Petch relationship for a magnesium alloy. Scripta Materialia 65, 994-997.

Zhang, J., Joshi, S., 2012. Phenomenological crystal plasticity modeling and detailed micromechanical investigations of pure magnesium. Journal of the Mechanics and Physics of Solids 60, 945-972. 


\section{Figure Captions}

Figure 1. Schematic showing average lattice orientation of extruded Mg.

Figure 2. Illustrations of Mg MMC unit cells indicating relative alignments of rod-shaped (a-b) and platelet-shaped (c-d) inclusions with reference to c-axis of $\mathrm{Mg}$ single crystal.

Figure 3. An example of sphere reinforced Mg MMC unit cell considered in this work. Similar unit cells are considered for rod-shaped and platelet-shaped inclusions.

Figure 4. True stress-true strain curves for different reinforcement shapes and alignments $\left(f_{r}=0.05\right)$.

Figure 5. Asymmetry ratio (AR) for Mg MMCs with different reinforcement shapes and alignments $\left(f_{r}=0.05\right)$.

Figure 6. Evolution of volume-averaged relative activities $\left(\bar{\Gamma}^{\alpha}\right)$ under compressive loading perpendicular to the c-axis.

Figure 7. Evolution of twinned regions under compressive loading perpendicular to the c-axis for (a-c) Rod-b, (d-f) Platelet-b and (g-i) Sphere Mg MMCs.

Figure 8. Evolution of extension twin volume fraction in $\mathrm{Mg}$ MMCs with different reinforcement shapes and alignments (compressive loading perpendicular to c-axis).

Figure 9. Relative activities of slip and twin modes under tensile loading for (a) Rod-b ,(b) Sphere,(c) Rod-c Mg MMCs and (d) Monolithic Mg.

Figure 10. Plot showing the volume fraction of the matrix with magnitude of hydrostatic stress $\left(\left|\sigma_{h}\right|\right)$ greater than the value indicated on the abscissa. The results pertain to a far-field tensile strain of $\varepsilon=0.05$ for tension loading perpendicular to the c-axis.

Figure 11. Accumulated plastic shear strain at the central sections for exemplar Mg MMCs corresponding to far-field tensile strain $\varepsilon=0.05$ for tension loading perpendicular to the c-axis. 
Figure 12. Effect of aspect ratio on tensile and compressive responses of Rod-b MMCs with fixed $f=0.05$

Figure 13. Effect of reinforcement volume fraction on the tension-compression asymmetry response of Rod-b Mg MMC. (Aspect ratio = 2). 
Figures

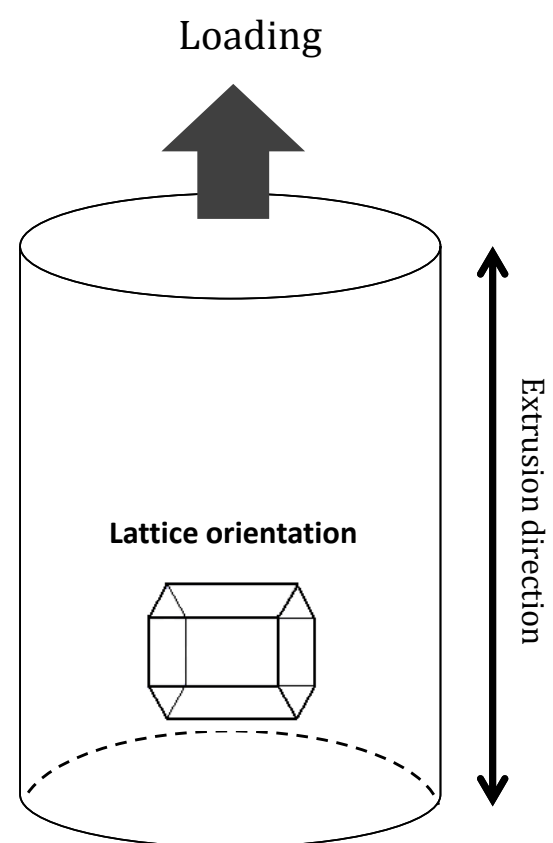

Figure 1 Schematic showing average lattice orientation of extruded Mg. 


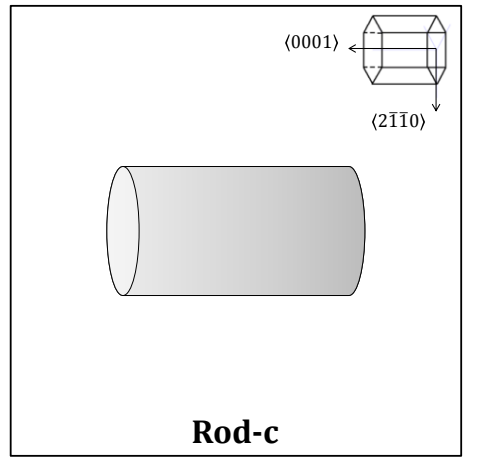

(a)

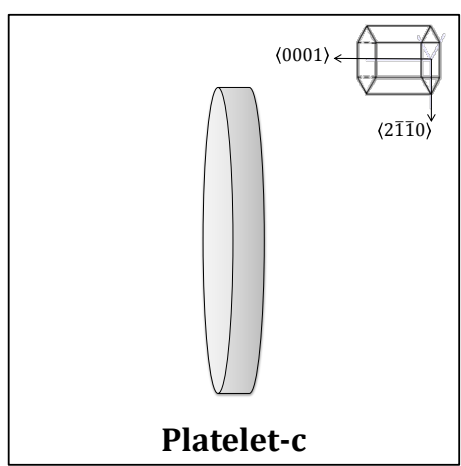

(c)

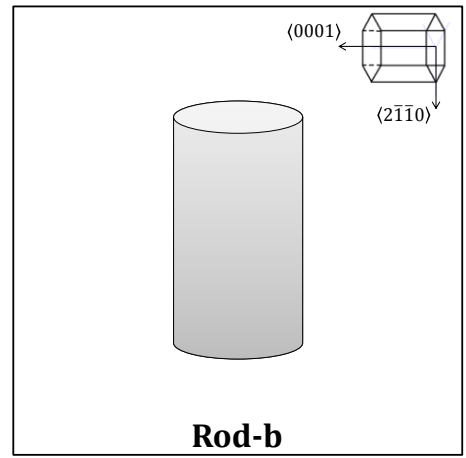

(b)

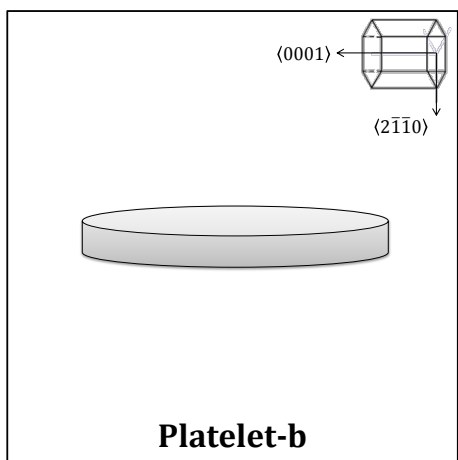

(d)

Figure 2 Illustrations of Mg MMC unit cells indicating relative alignments of rod-shaped (a-b) and platelet-shaped (c-d) inclusions with reference to c-axis of $\mathrm{Mg}$ single crystal. 


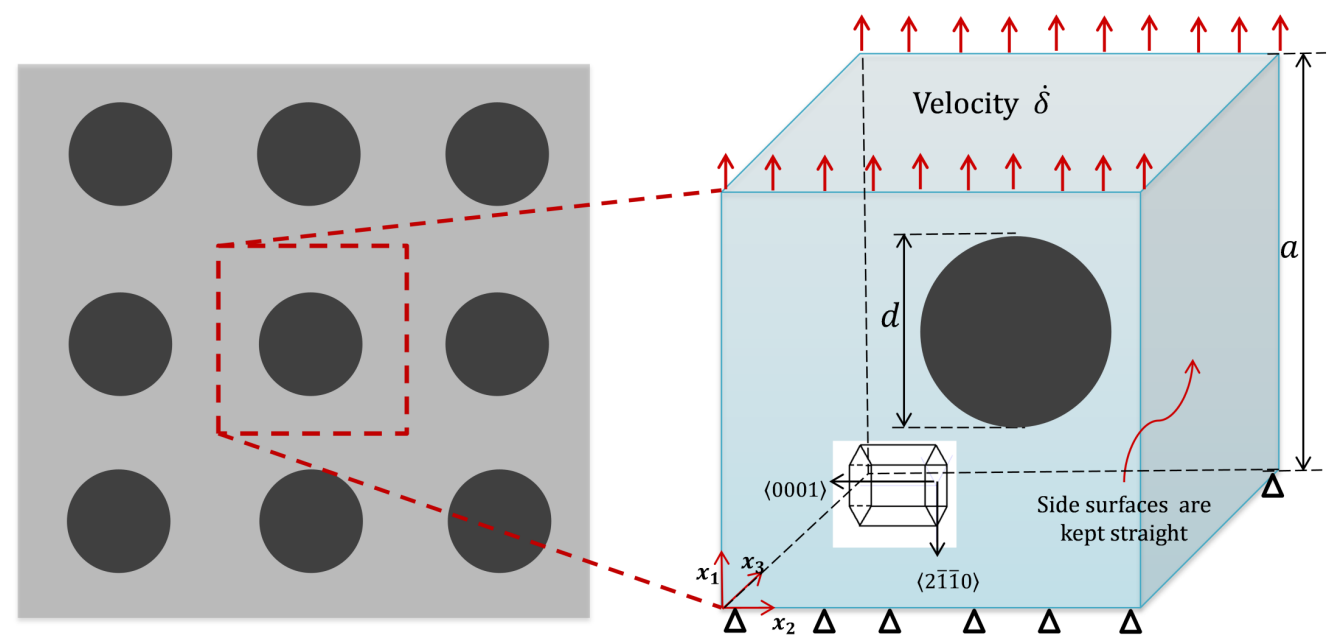

Figure 3 An example of sphere reinforced Mg MMC unit cell considered in this work. Similar unit cells are considered for rod-shaped and platelet-shaped inclusions. 


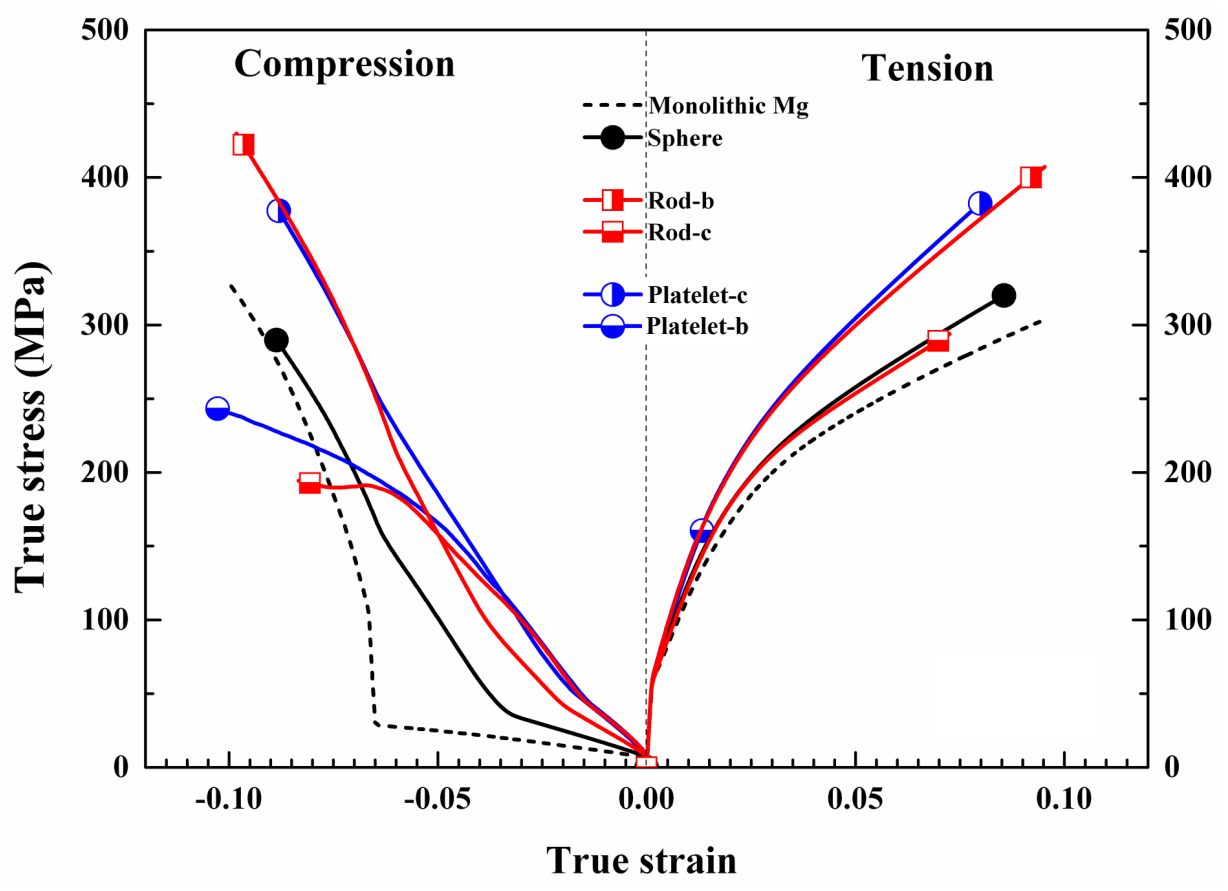

Figure 4 True stress-true strain curves for different reinforcement shapes and alignments $\left(f_{r}=0.05\right)$. 


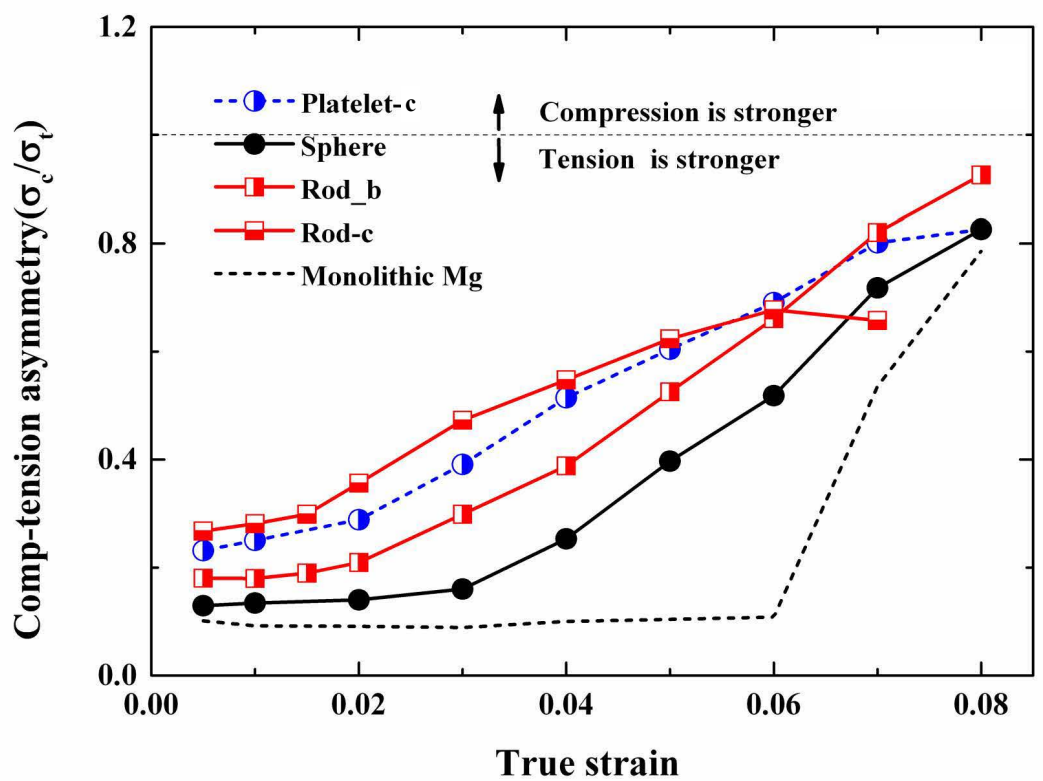

Figure 5 Asymmetry ratio (AR) for Mg MMCs with different reinforcement shapes and alignments $\left(f_{r}=0.05\right)$. 


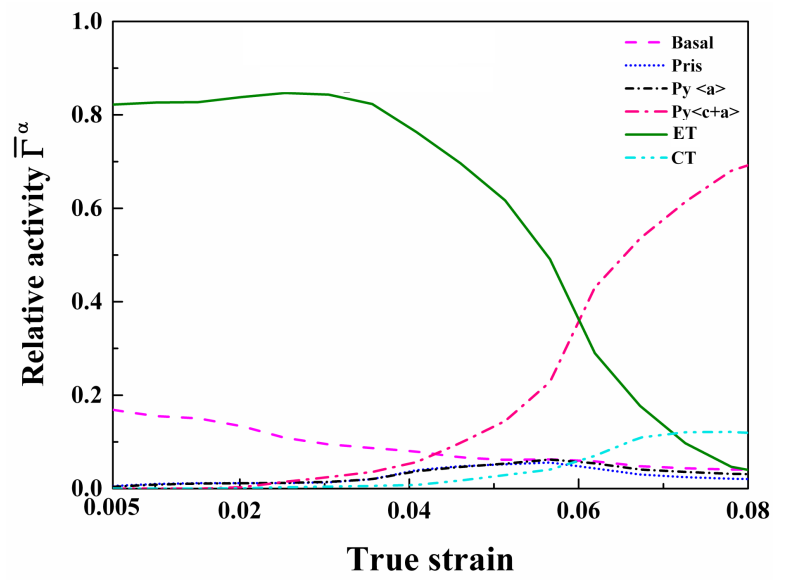

(a) Rod-b

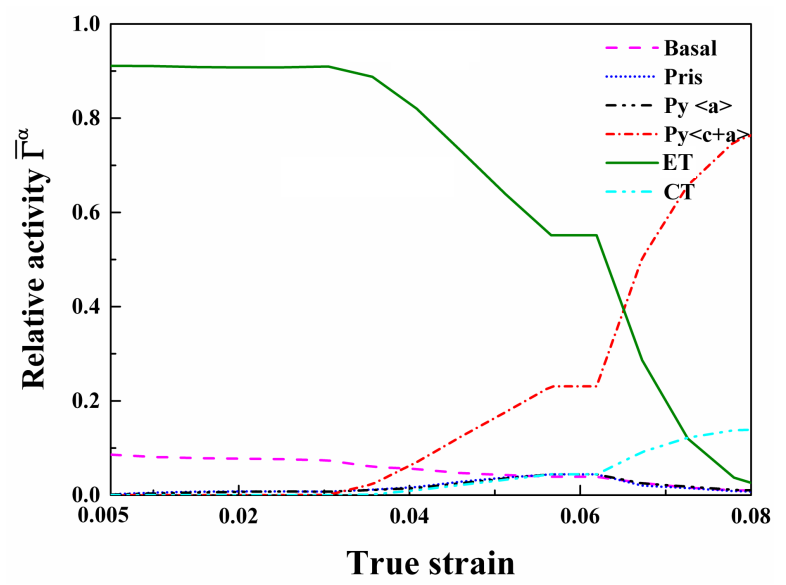

(c) Sphere

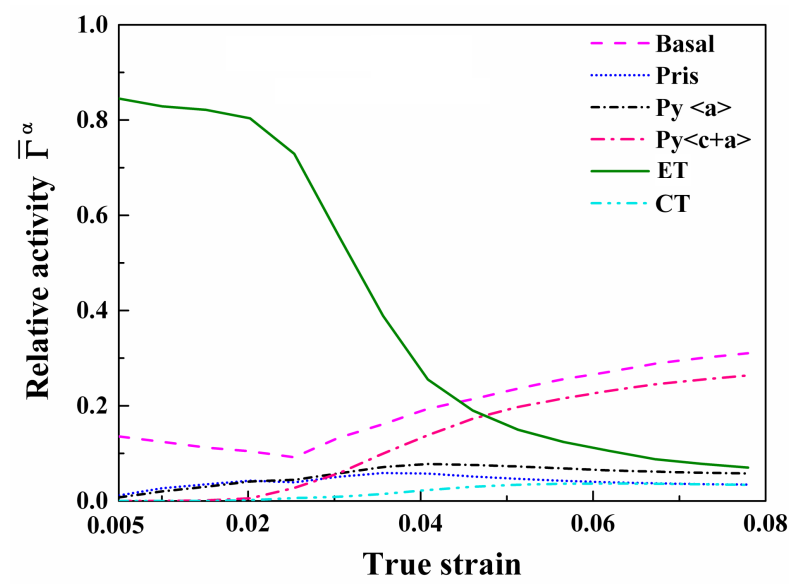

(b) Platelet-b

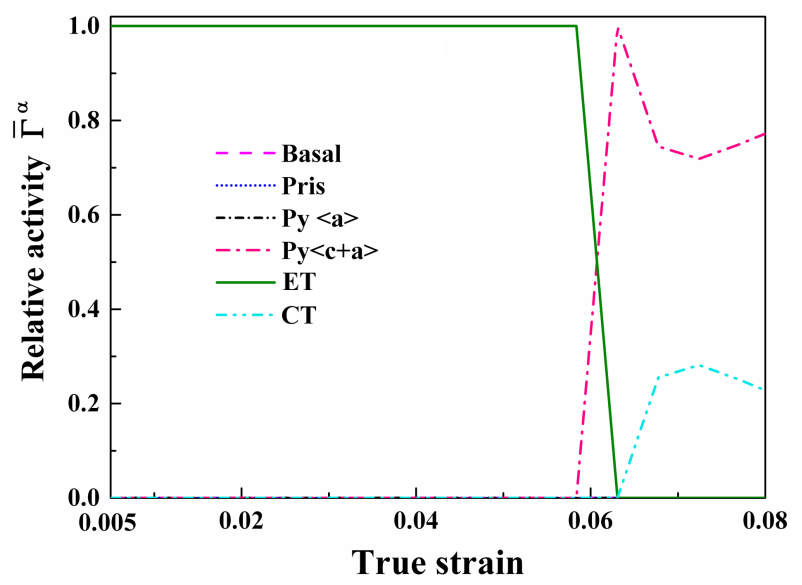

(d) Monolithic Mg

Figure 6 Evolution of volume-averaged relative activities $\left(\bar{\Gamma}^{\alpha}\right)$ under compressive loading perpendicular to the c-axis. 


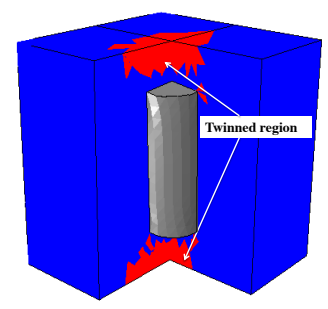

(a) $\bar{\varepsilon}=0.03$

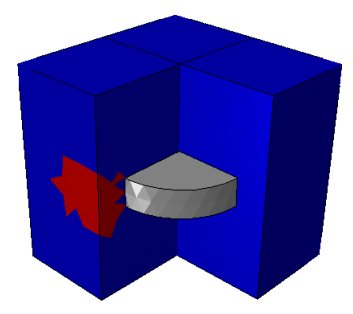

(d) $\bar{\varepsilon}=0.03$

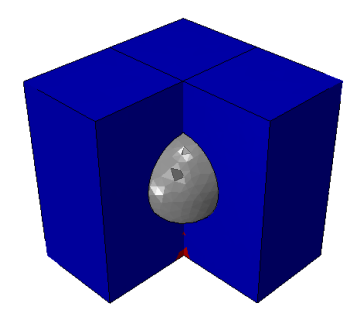

(g) $\bar{\varepsilon}=0.03$

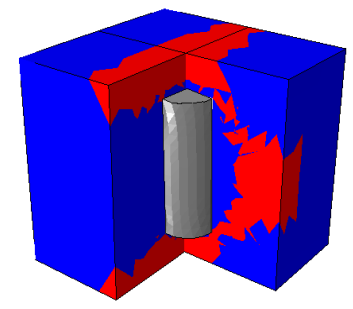

(b) $\bar{\varepsilon}=0.04$

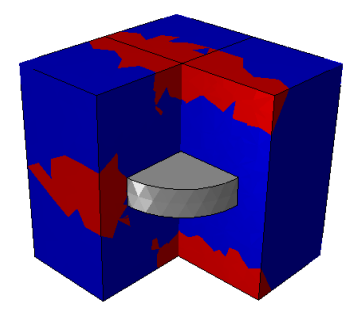

(e) $\bar{\varepsilon}=0.04$

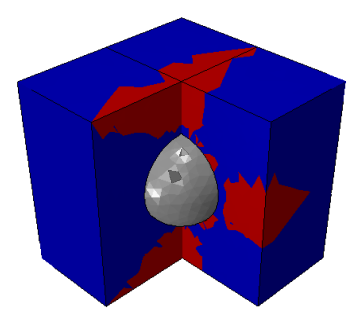

(h) $\bar{\varepsilon}=0.04$

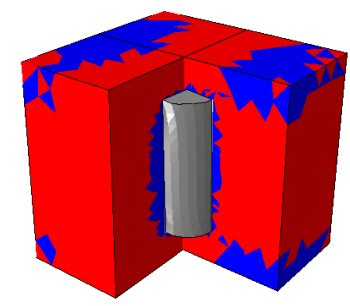

(c) $\bar{\varepsilon}=0.07$

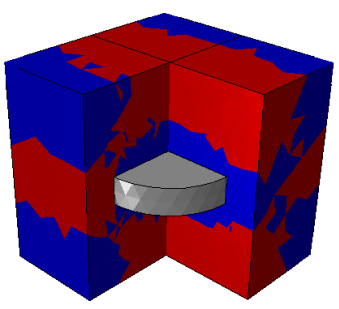

(f) $\bar{\varepsilon}=0.07$

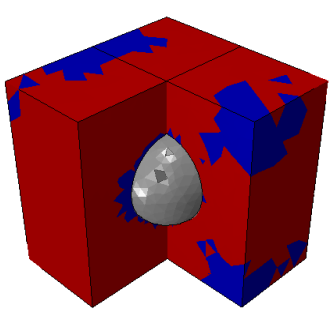

(i) $\bar{\varepsilon}=0.07$

Figure 7 Evolution of twinned regions under compressive loading perpendicular to the c-axis for (a-c) Rod-b, (d-f) Platelet-b and (g-i) Sphere Mg MMCs. 


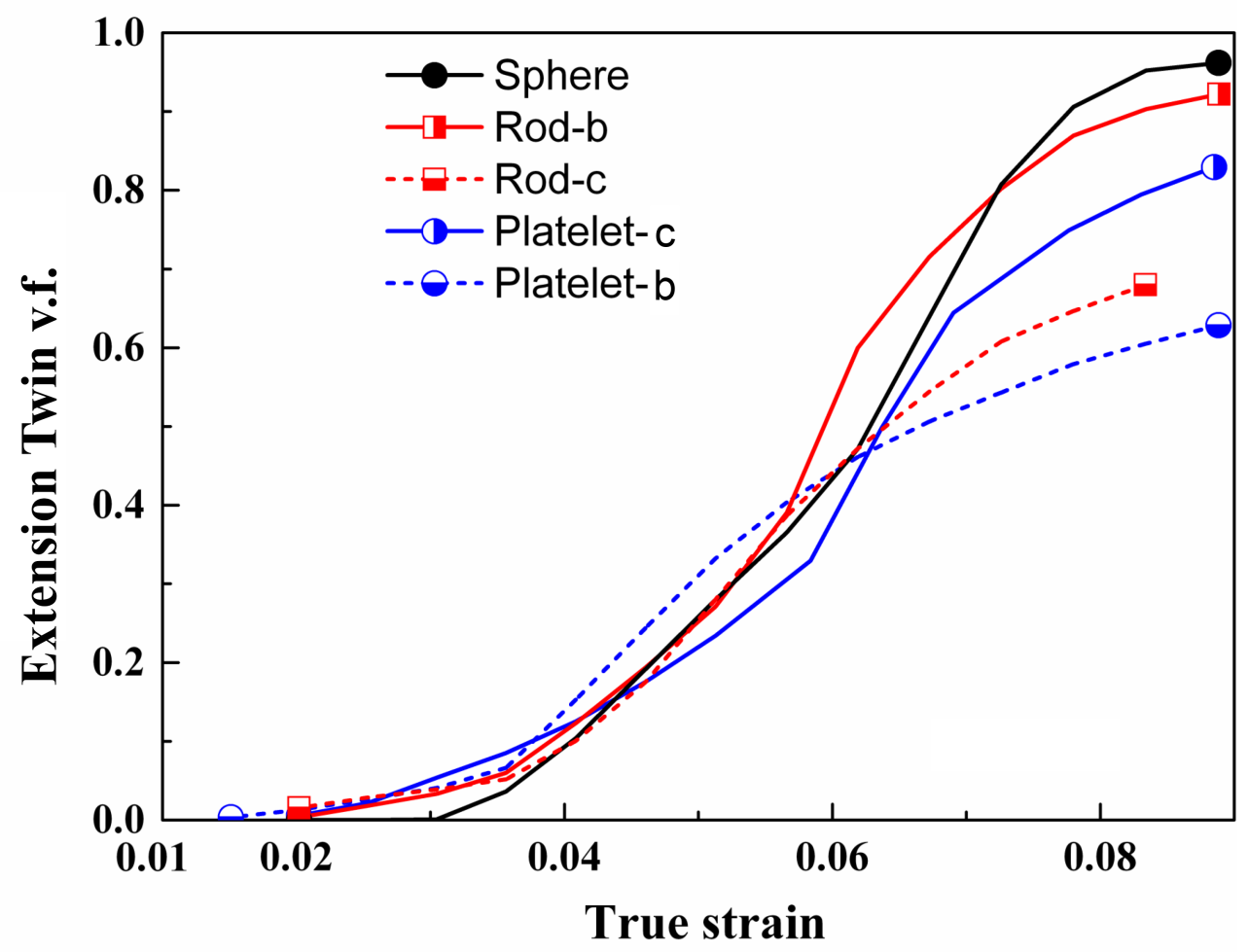

Figure 8 Evolution of extension twin volume fraction in Mg MMCs with different reinforcement shapes and alignments (compressive loading perpendicular to c-axis). 


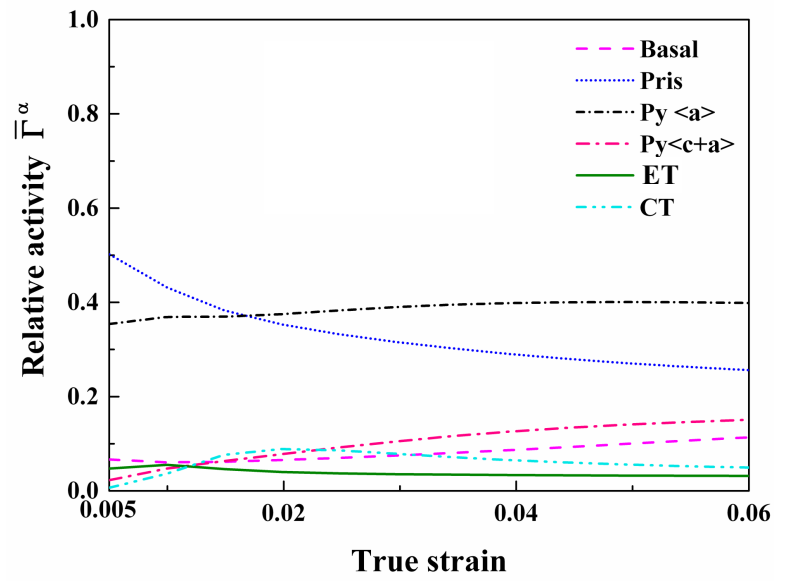

(a) Rod-b

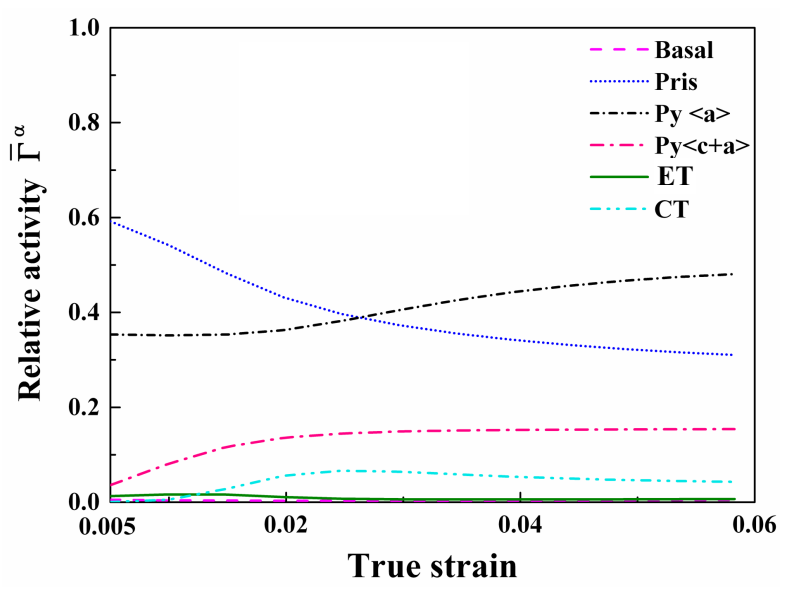

(c) Rod-c

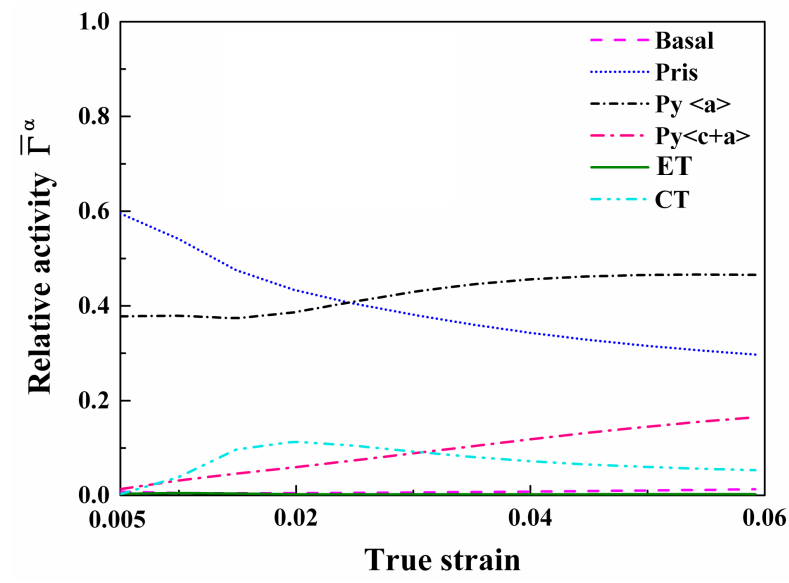

(b) Sphere

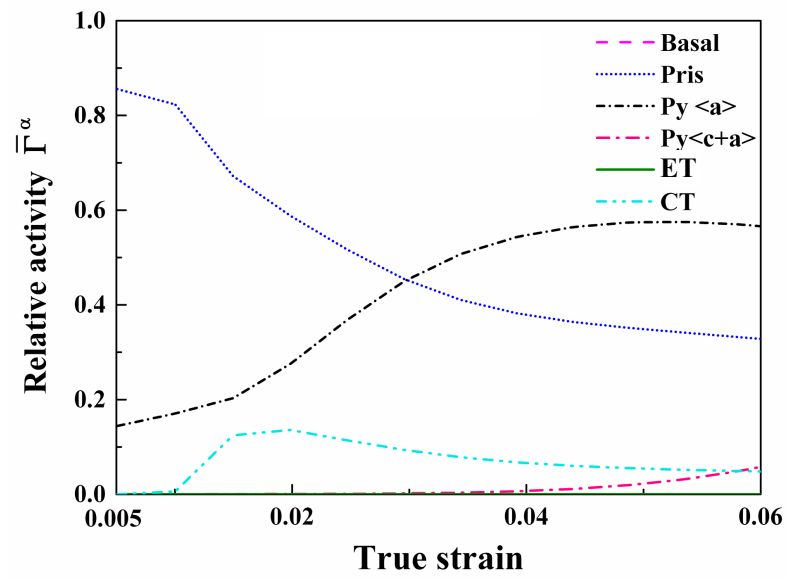

(d) Monolithic Mg

Figure 9 Relative activities of slip and twin modes under tensile loading for (a) Rod-b ,(b) Sphere, (c) Rod-c Mg MMCs and (d) Monolithic Mg. 


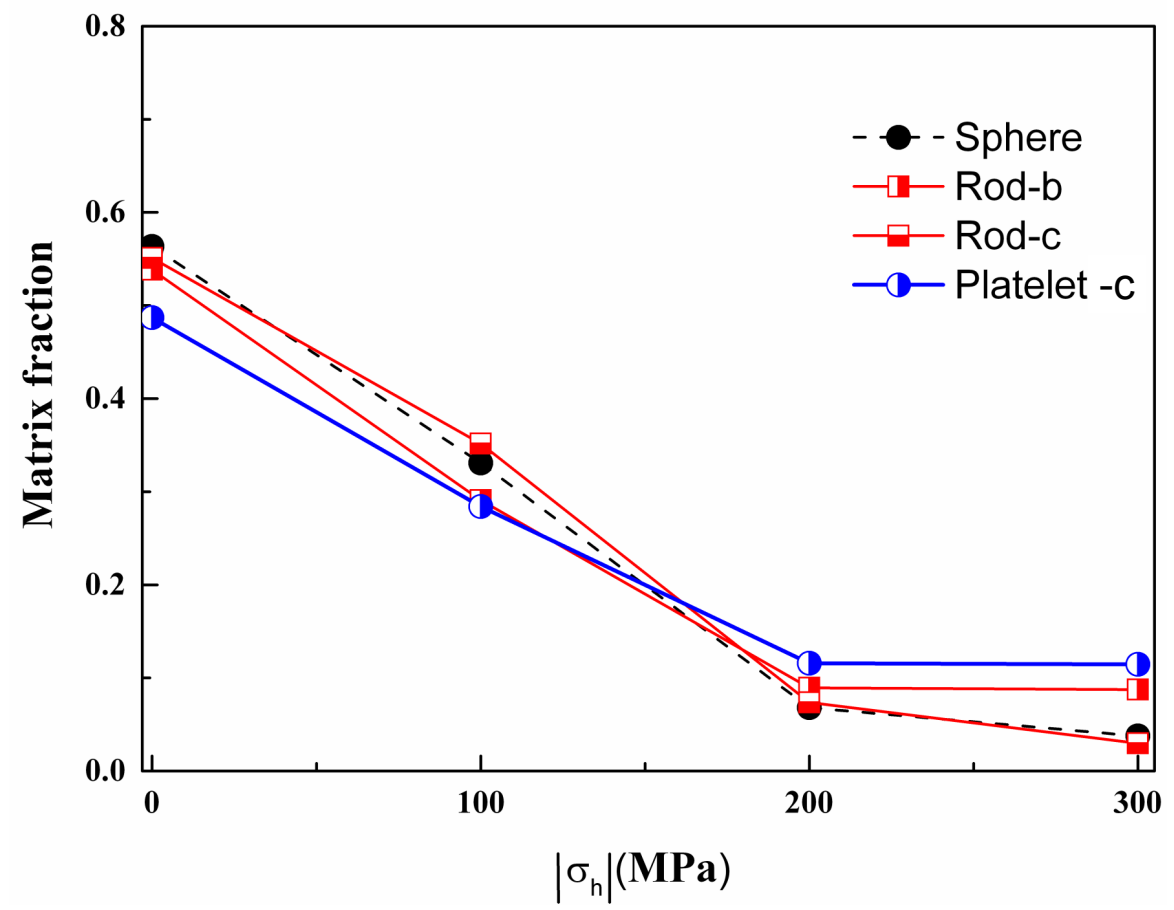

Figure 10 Plot showing the volume fraction of the matrix with magnitude of hydrostatic stress $\left(\left|\sigma_{h}\right|\right)$ greater than the value indicated on the abscissa. The results pertain to a far-field tensile strain of $\varepsilon=0.05$ for tension loading perpendicular to the c-axis. 


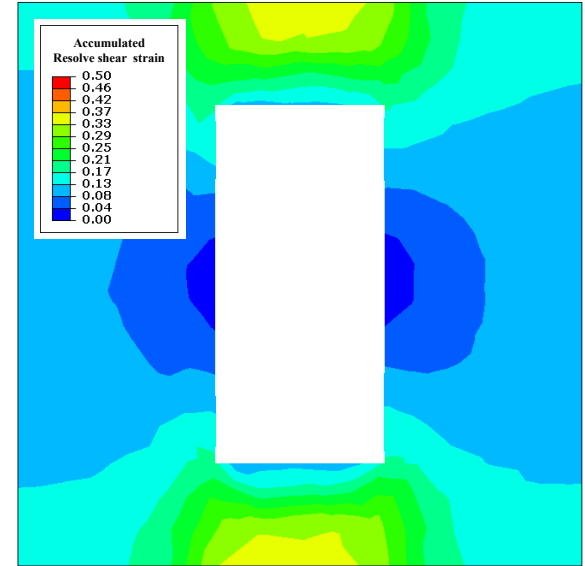

(a) Rod-b

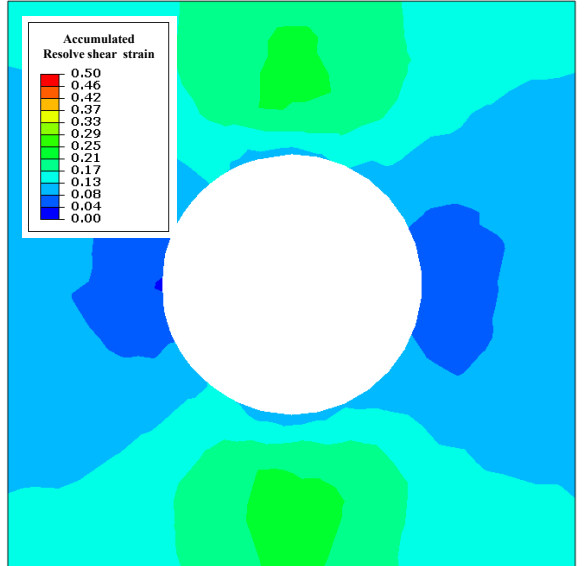

(b) Sphere

Figure 11 Accumulated plastic shear strain at the central sections for exemplar Mg MMCs corresponding to far-field tensile strain $\varepsilon=0.05$ for tension loading perpendicular to the c-axis. 


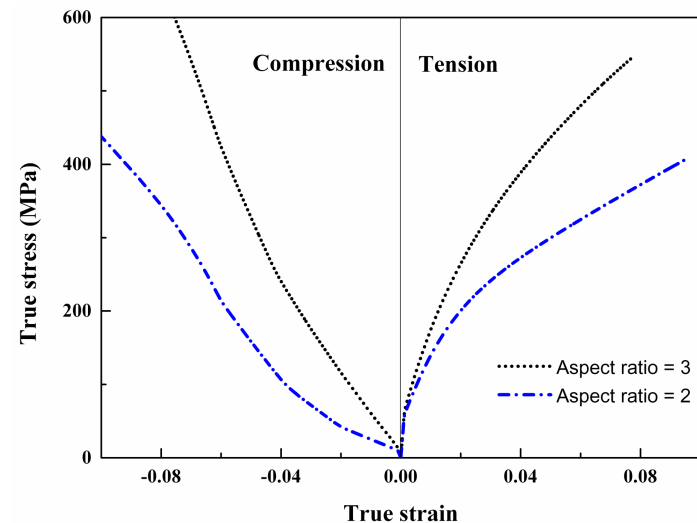

(a)

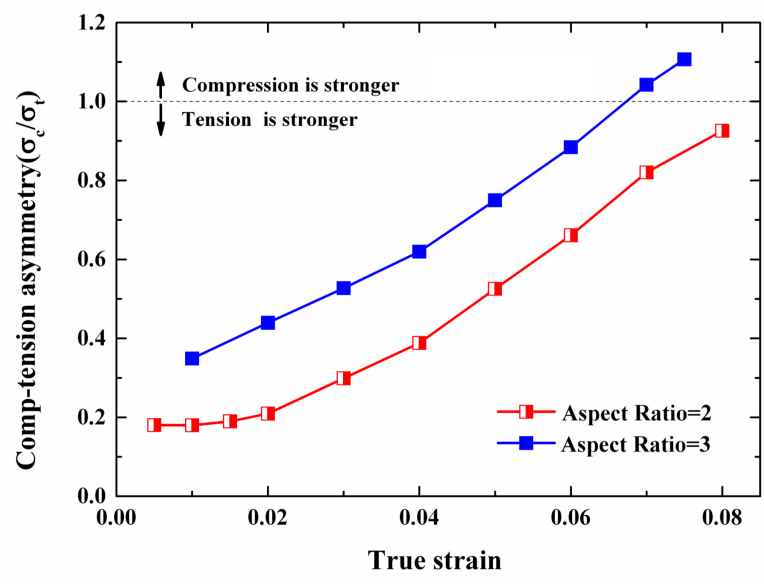

(b)

Figure 12 Effect of aspect ratio on tensile and compressive responses of Rod-b MMCs with fixed $f=0.05$ 


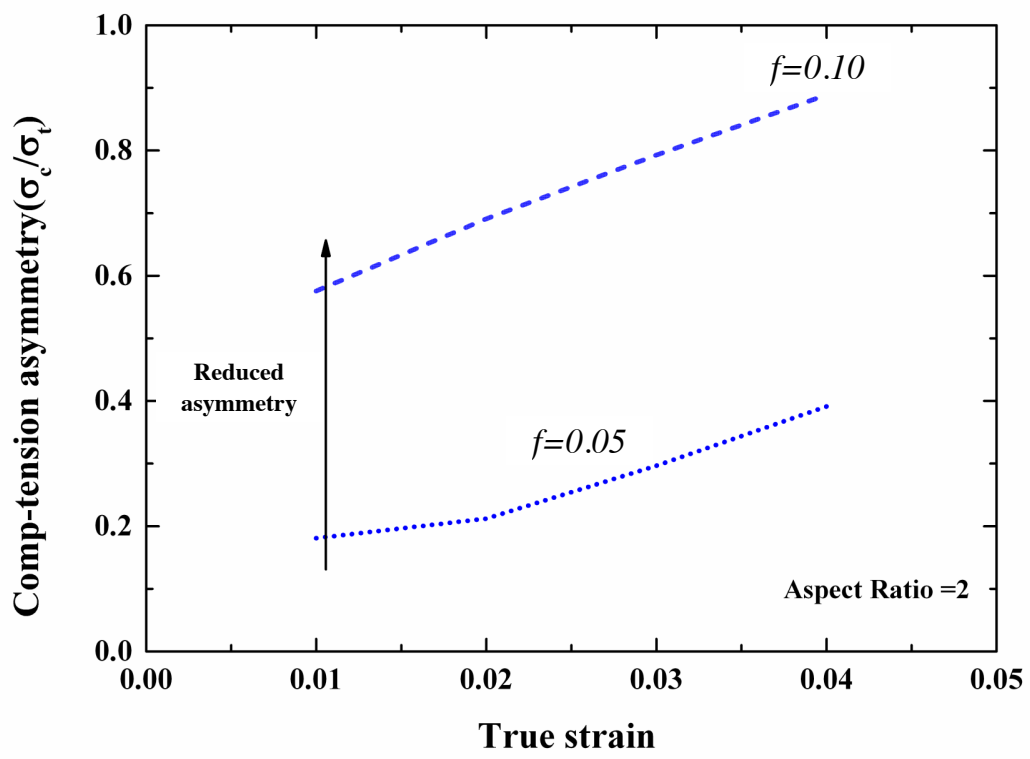

Figure 13 Effect of reinforcement volume fraction on the tension-compression asymmetry response of Rod- $b$ Mg MMC. (Aspect ratio = 2). 


\section{Tables}

Table 1 Slip and twin modes in Mg matrix (Zhang and Joshi, 2012)

\begin{tabular}{lccc}
\hline Mode & Type & Number of systems & Normalized CRSS $\left(\tau / \tau_{\text {Basal }}\right)$ \\
\hline Basal $\langle a\rangle$ & $(0001)\langle 11 \overline{2} 0\rangle$ & 3 & 1 \\
Prismatic $\langle a\rangle$ & $\{10 \overline{1} 0\}\langle 11 \overline{2} 0\rangle$ & 3 & 50 \\
Pyramidal $\langle a\rangle$ & $\{10 \overline{1} 1\}\langle 11 \overline{2} 0\rangle$ & 6 & 50 \\
Pyramidal $\langle c+a\rangle$ & $\{11 \overline{2} 2\}\langle 11 \overline{2} 3\rangle$ & 6 & 80 \\
Tension twin & $\{10 \overline{1} 2\}\langle 10 \overline{1} 1\rangle$ & 6 & 7 \\
Compression twin & $\{10 \overline{1} 1\}\langle 10 \overline{1} \overline{2}\rangle$ & 6 & 110 \\
\hline
\end{tabular}


Table 2 Material parameters for pure $\mathrm{Mg}$

\begin{tabular}{|c|c|c|c|c|}
\hline & $\tau_{0}(\mathrm{MPa})$ & $h_{\circ}(\mathrm{MPa})$ & $\tau_{s}(\mathrm{MPa})$ & \\
\hline Basal Slip & 0.5 & 20 & - & \\
\hline Prismatic $\langle a\rangle$ Slip & 25 & 1500 & 85 & \\
\hline Pyramidal $\langle a\rangle$ Slip & 25 & 1500 & 85 & \\
\hline \multirow[t]{2}{*}{ Pyramidal $\langle c+a\rangle$ Slip } & 40 & 3000 & 150 & \\
\hline & $\tau_{0}(\mathrm{MPa})$ & $h_{e t}(\mathrm{MPa})$ & $\tau_{s \_e t}(\mathrm{MPa})$ & $h_{e t \_s l}(\mathrm{MPa})$ \\
\hline \multirow[t]{2}{*}{ Extension Twinning } & 3.5 & 100 & 20 & 100 \\
\hline & $\tau_{0}(\mathrm{MPa})$ & $H_{c t}(\mathrm{MPa})$ & $H_{c t \_s l}(\mathrm{MPa})$ & $b$ \\
\hline Contraction Twinning & 55 & 6000 & 15 & 0.05 \\
\hline
\end{tabular}


Table 3 Correlation between twin volume fraction average hydrostatic stress and flow stress in Mg MMC under compressive loading.

\begin{tabular}{lllll}
\hline Reinforcement & Nominal & Extension & Average & hydro- \\
type & strain & Average & twin volume \\
& & static stress & Stress \\
& $\bar{\varepsilon}$ & $\xi_{\mathrm{t}}$ & $\bar{\sigma}_{\mathrm{H}}(\mathrm{MPa})$ & $\bar{\sigma}_{\mathrm{f}}(\mathrm{MPa})$ \\
\hline Platelet-c & & 0.04 & 45.4 & 104 \\
Rod-c & & 0.04 & 42.4 & 101 \\
Platelet-b & 0.03 & 0.05 & 32.7 & 98 \\
Rod-b & & 0.03 & 17 & 73 \\
Sphere & & 0.004 & 10.7 & 34 \\
\hline Rod-b & & 0.75 & 81.4 & 287 \\
Platelet-b & & 0.66 & 94.9 & 286 \\
Platelet-c & 0.07 & 0.52 & 97.6 & 204 \\
Rod-c & & 0.57 & 76.4 & 202 \\
Sphere & & 0.71 & 72.1 & 191 \\
\hline
\end{tabular}


Table 4 Contribution from constraint effect and twinning to the overall compressive flow stress at $\bar{\varepsilon}=0.07$ (compressive loading perpendicular to c-axis).

\begin{tabular}{|c|c|c|c|c|}
\hline $\begin{array}{l}\text { Type } \\
\text { (MPa) }\end{array}$ & $\xi_{\mathrm{t}}$ & $\bar{\sigma}_{\mathrm{H}}^{\prime}$ & $\begin{array}{l}\bar{\sigma}_{\mathrm{f}} \\
(\mathrm{MPa})\end{array}$ & $\begin{array}{l}A_{\mathrm{avg}} \xi_{\mathrm{t}} \bar{\sigma}_{\mathrm{H}}^{\prime} \\
(\mathrm{MPa})\end{array}$ \\
\hline Rod-b & 0.75 & 1610 & 287 & 304.3 \\
\hline Platelet-b & 0.66 & 1555 & 286 & 258.6 \\
\hline Platelet-c & 0.52 & 1623 & 204 & 212.6 \\
\hline Sphere & 0.71 & 1378 & 202 & 197.9 \\
\hline Rod-c & 0.57 & 1093 & 191 & 195.4 \\
\hline
\end{tabular}


Table 5 Relative activities of slip and twin modes for Mg MMCs $(f=0.05)$ Sphere and Rod-b Mg MMC at $\bar{\varepsilon}=0.005$

\begin{tabular}{cccccccc}
\hline Reinforcement shape & $\bar{\Gamma}^{\alpha}$ & Basal $\langle a\rangle$ & Pris $\langle a\rangle$ & $\operatorname{Py}\langle a\rangle$ & $\operatorname{Py}\langle c+a\rangle$ & ET & CT \\
\hline \multirow{2}{*}{ Sphere } & Compression & 0.17 & 0.0 & 0.0 & 0.0 & 0.83 & 0.0 \\
& Tension & 0 & 0.75 & 0.2 & 0.01 & 0.04 & 0.0 \\
\hline \multirow{2}{*}{ Rod-b } & Compression & 0.17 & 0.01 & 0.0 & 0.0 & 0.82 & 0 \\
& Tension & 0.04 & 0.30 & 0.43 & 0.15 & 0.01 & 0.06 \\
\hline
\end{tabular}


Table 6 Correlation between compression-tension asymmetry and $\bar{\Pi}$ for Sphere and Rod-b Mg MMCs

\begin{tabular}{cccccc}
\hline Reinforcement shape & Loading & $0.5 \%$ yield stress & $\sum \bar{\Gamma}^{\alpha} \tau_{\alpha}$ & $\Pi$ & $\bar{\Pi}$ \\
\hline \multirow{2}{*}{ Sphere } & Compression & 11 & 3.0 & 3.7 & \\
& Tension & 85 & 24.3 & 3.5 & \\
\hline \multirow{2}{*}{ Rod-b } & compression & 17 & 3.21 & 5.3 & \\
& Tension & 96.5 & 27.6 & 3.5 & \\
\hline
\end{tabular}

